\title{
Whey protein-derived exosomes increase protein synthesis and hypertrophy in $\mathrm{C}_{2} \mathrm{C}_{12}$ myotubes
}

\author{
C. Brooks Mobley, ${ }^{*}$ Petey W. Mumford, ${ }^{\star}$ John J. McCarthy,† Michael E. Miller, $\ddagger$ Kaelin C. Young, ${ }^{*} \S$ \\ Jeffrey S. Martin, ${ }^{\star} \S$ Darren T. Beck, ${ }^{\star} \S$ Christopher M. Lockwood,\# and Michael D. Roberts ${ }^{\star}{ }^{1}$ \\ *School of Kinesiology, Auburn University, Auburn, AL 36849 \\ †Department of Physiology, University of Kentucky Medical College of Medicine, Lexington 40506 \\ łHarrison School of Pharmacy, Auburn University, Auburn, AL 36849 \\ $\S$ Department of Cellular Biology and Physiology, Edward Via College of Osteopathic Medicine, Auburn Campus, Auburn, AL 36849 \\ \#AP Nutrition LLC, Draper, UT 84020
}

\section{ABSTRACT}

We sought to examine potential amino acid independent mechanisms whereby hydrolyzed whey protein (WP) affects muscle protein synthesis (MPS) and anabolism in vitro. Specifically, we tested (1) whether 3-h and 6-h treatments of WP, essential amino acids, or L-leucine (Leu) affected MPS, and whether 6-h treatments with low-, medium-, or high doses of WP versus Leu affected MPS; (2) whether knockdown of the primary Leu transporter affected WP- and Leu-mediated changes in MPS, mammalian target of rapamycin (mTOR) signaling responses, or both, following 6-h treatments; (3) whether exosomes isolated from WP (WP-EXO) affected MPS, mTOR signaling responses, or both, compared with untreated (control) myotubes, following 6 -h, 12-h, and 24-h treatments, and whether they affected myotube diameter following $24-\mathrm{h}$ and $48-\mathrm{h}$ treatments. For all treatments, 7-d post-differentiated $\mathrm{C}_{2} \mathrm{C}_{12}$ myotubes were examined. In experiment $1,6-\mathrm{h}$ WP treatments increased MPS compared with control $(+46 \%)$, Leu $(+24 \%)$, and essential amino acids $(+25 \%)$. Moreover, the 6-h low-, medium-, and high WP treatments increased MPS by approximately 40 to $50 \%$ more than corresponding Leu treatments. In experiment 2 (LAT short hairpin RNA-transfected myotubes), 6-h WP treatments increased MPS compared with control $(+18 \%)$ and Leu $(+19 \%)$. In experiment 3 , WP-EXO treatments increased MPS over controls at $12 \mathrm{~h}(+18 \%)$ and $24 \mathrm{~h}(+45 \%)$, and myotube diameters increased with $24-\mathrm{h}(+24 \%)$ and $48-\mathrm{h}(+40 \%)$ WP-EXO treatments compared with controls. The WP-EXO treatments did not appear to operate through mTOR signaling; instead, they increased mRNA and protein levels o eukaryotic initiation factor 4A. Bovine-specific

Received April 20, 2016.

Accepted September 1, 2016.

${ }^{1}$ Corresponding author: mdr0024@auburn.edu
microRNA following 24-h WP-EXO treatments were enriched in myotubes (chiefly miR-149-3p, miR-2881), but were not related to hypertrophic gene targets. To summarize, hydrolyzed WP-EXO increased skeletal MPS and anabolism in vitro, and this may be related to an unknown mechanism that increases translation initiation factors rather than enhancing mTOR signaling or the involvement of bovine-specific microRNA.

Key words: muscle protein synthesis, whey protein, exosomes, leucine

\section{INTRODUCTION}

The mammalian target of rapamycin (mTOR) signaling pathway is central in the regulation of skeletal muscle protein synthesis (MPS). Various studies (Stitt et al., 2004; Latres et al., 2005) have successfully used the murine $\mathrm{C}_{2} \mathrm{C}_{12}$ skeletal muscle cell culture model to evaluate the effects of amino acids on mTOR signaling, and amino acids have been deemed pivotal in regulating multiple mTOR intermediaries, such as p70s6k, rpS6, 4E-BP1, eIF4E, eIF4G, and eEF2 (Wang et al., 1998; Kimball et al., 1999; Peyrollier et al., 2000). Importantly, L-leucine (Leu) is unique among amino acids due to its ability to profoundly increase mTOR pathway activation (Atherton et al., 2010) and increase MPS (Deldicque et al., 2008).

Although Leu is a well-established stimulator of MPS, whey protein (WP) also robustly increases MPS. Whey protein contains a high concentration of Leu (Hulmi et al., 2010; van Vliet et al., 2015) and possesses an amino acid composition that resembles that of skeletal muscle (Ha and Zemel, 2003; Burd et al., 2013). Such attributes may favor muscle growth when comparing WP to single amino acids (van Vliet et al., 2015). As well, WP contains other biological components that confer unique physiological effects. For instance, we recently reported that WP feedings immediately following an acute bout of exercise promoted increases in post- 
exercise MPS in rodents compared with Leu only (Mobley et al., 2016). Moreover, 2 of our previous rodent studies have demonstrated that acute hydrolyzed WP feedings in rodents increased lipolysis markers in serum (Roberts et al., 2014) and adipose tissue (Mobley et al., 2015) compared with other protein forms (i.e., native WP concentrate and egg albumin, respectively). With regard to potential bioactives in WP, exosomes from bovine milk (Izumi et al., 2012; Reinhardt et al., 2012; Yamada et al., 2012; Izumi et al., 2015) and WP (Izumi et al., 2013) have been isolated and identified, and these vesicles contain bioactive components, including peptides, mRNA, and microRNA (miRNA; Chen et al., 2010; Hata et al., 2010). Recently, it has been reported that bovine milk-derived exosomes interact with human peripheral blood mononuclear cells (Baier et al., 2014), human embryonic kidney 293 (HEK293) cells (Baier et al., 2014), and human macrophages (Izumi et al., 2015) to affect gene expression in various cell types. Although no study to our knowledge has examined how bovinederived exosomes affect skeletal muscle anabolism, it has been posited that bovine and WP-derived exosomes (WP-EXO) possess miRNA (e.g., miR21 and miR29a) that may affect gene targets and, in turn, may amplify mTOR pathway-related targets and enhance MPS (Melnik et al., 2013; Melnik, 2015).

The purpose of this study was to identify possible mechanisms, beyond essential amino acid (EAA) and Leu-mediated signaling, by which WP may enhance protein synthesis in $\mathrm{C}_{2} \mathrm{C}_{12}$ myotubes. The aims of these experiments were as follows: (1) to compare hydrolyzed WP with EAA and Leu on MPS levels in $\mathrm{C}_{2} \mathrm{C}_{12}$ myotubes; (2) to examine whether hydrolyzed WP increases MPS in $\mathrm{C}_{2} \mathrm{C}_{12}$ myotubes following knockdown of the primary Leu transporter (LAT1); and (3) examine whether exosomes derived from hydrolyzed WP affect MPS and hypertrophy in $\mathrm{C}_{2} \mathrm{C}_{12}$ myotubes, and whether this is associated with mTOR signaling, the appearance of bovine-specific miRNA related to muscle anabolism, or both.

\section{MATERIALS AND METHODS}

\section{Cell Culture Methods}

For all experiments, $\mathrm{C}_{2} \mathrm{C}_{12}$ myoblasts, passage no. $5-15$, were maintained in growth medium [Dulbecco's Modified Eagle Medium (DMEM), 10\% fetal bovine serum, $1 \%$ penicillin/streptomycin, $0.1 \%$ gentamycin) under standard conditions at $37^{\circ} \mathrm{C}$ in a $5 \% \mathrm{CO}_{2}$ atmosphere. Myoblasts were seeded on 6-well plates (Griener Bio-One GmbH, Frickenhausen, Germany) at a density of $3 \times 10^{5}$ in $3 \mathrm{~mL} /$ well of growth medium for protein and mRNA analyses. Differentiation was induced 48 $\mathrm{h}$ after myoblast growth reached 80-90\% confluency by removing the growth medium and replacing it with differentiation medium [DMEM, 2\% (vol/vol) horse serum, $1 \%$ penicillin/streptomycin, $0.1 \%$ gentamycin]. The differentiation medium was then replaced every 24 $\mathrm{h}$ for $7 \mathrm{~d}$ while treatments occurred.

\section{Experiment 1}

Effects of EAA, Leu, and WP on MPS. $\mathrm{C}_{2} \mathrm{C}_{12}$ myoblasts were seeded in 6 -well plates and differentiated as described above. Following $7 \mathrm{~d}$ of differentiation, myotubes were treated acutely with 1 of the following treatments for 3 or $6 \mathrm{~h}$ : (1) HyClone minimum Essential medium (MEM) only (GE Healthcare, Logan, $\mathrm{UT}$ ); (2) MEM and EAA [75 $\mu \mathrm{g} / \mathrm{mL}$ L-histidine (MP Biomedicals, Solon, OH), $240 \mu \mathrm{g} / \mathrm{mL}$ L-isoleucine (Affymetrix Inc., Cleveland, OH), $750 \mu \mathrm{g} / \mathrm{mL}$ L-leucine (EMD Chemicals, San Diego, CA), $360 \mu \mathrm{g} / \mathrm{mL}$ L-lysine (TCI Ltd., Toshima, Kita-Ku, Tokyo, Japan), $85 \mu \mathrm{g} /$ $\mathrm{mL}$ L-methionine (Ameresco, Solon, OH), $130 \mu \mathrm{g} /$ $\mathrm{mL}$ L-phenylalanine (MP Biomedicals), $250 \mu \mathrm{g} / \mathrm{mL} \mathrm{L-}$ threonine (Ameresco), $250 \mu \mathrm{g} / \mathrm{mL}$ L-tryptophan (Alfa Aesar, Ward Hill, MA), and $65 \mu \mathrm{g} / \mathrm{mL}$ L-valine (Alfa Aesar); the EAA content was matched to the EAA profile of the WP source; see Table 1]; (3) MEM and 750 $\mu \mathrm{g} / \mathrm{mL}$ Leu (EMD Chemicals); or (4) MEM and $5 \mathrm{mg} /$ mL hydrolyzed WP (Hilmar Ingredients, Hilmar, CA). The amount of Leu to use for treatment was based on previous literature (Hulmi et al., 2010) suggesting that 12 to $15 \%$ of WP is Leu; moreover, the Leu content was similar to that in the WP treatments. Approximately 30 min before cell lysis, cells were pulse-labeled with 1 $\mu M$ puromycin hydrochloride (Ameresco) in PBS for subsequent MPS assessment using the surface sensing of translation method as performed in our laboratory (Mobley et al., 2014) and other laboratories (Stec et al., 2016).

Table 1. Essential amino acid profile of whey protein source

\begin{tabular}{lc}
\hline Amino acid & $\mathrm{g} / 100 \mathrm{~g}$ of powder \\
\hline Histidine $^{1}$ & 1.5 \\
Isoleucine $^{1}$ & 4.8 \\
Leucine $^{1}$ & 9.0 \\
Lysine $^{\text {Methionine }}$ & 7.2 \\
Phenylalanine & 1.7 \\
Threonine & 2.6 \\
Tryptophan $_{\text {Valine }}{ }^{1}$ & 5.0 \\
\end{tabular}

${ }^{1}$ Branched-chain amino acids. 
Following puromycin pulse-labeling, cells were lysed using cell lysis buffer (Tris base; $\mathrm{pH}$ 8.0, NaCl, NP-40, sodium deoxycholate, SDS; Cell Signaling Technology, Danvers, MA) containing protease and phosphatase inhibitors (G-Biosciences, St. Louis, MO). After cells were lysed, each plate was scraped using a $25-\mathrm{cm}$ cell scraper (VWR, Radnor, PA), homogenates were centrifuged at $500 \times g$ for $5 \mathrm{~min}$, and supernatants were analyzed for total protein using a bicinchoninic acid (BCA) assay (Pierce, Rockford, IL). Supernatants were then stored at $-20^{\circ} \mathrm{C}$ for Western blotting analyses as described below.

Effects of Low, Medium, and High Doses of Leu or WP on MPS. A separate batch of $\mathrm{C}_{2} \mathrm{C}_{12}$ myoblasts were seeded in 6 -well plates and differentiated as described above. Following $7 \mathrm{~d}$ of differentiation, myotubes were treated acutely with 1 of the following treatments for $6 \mathrm{~h}$ : (1) Leu-free DMEM only (Crystalgen, Commack, NY); (2) Leu-free DMEM and 150 $\mu \mathrm{g} / \mathrm{mL}$ (low dose), $750 \mu \mathrm{g} / \mathrm{mL}$ (medium dose), or 1.5 $\mathrm{mg} / \mathrm{mL}$ (high dose) Leu (EMD Chemicals); or (3) 1 $\mathrm{mg} / \mathrm{mL}$ (low dose), $5 \mathrm{mg} / \mathrm{mL}$ (medium dose), or 10 $\mathrm{mg} / \mathrm{mL}$ (high dose) of hydrolyzed WP (Hilmar Ingredients). The Leu content in each Leu treatment dose was slightly higher than the Leu present in the WP treatments. Approximately $30 \mathrm{~min}$ before cell lysis, cells were pulse-labeled with $1 \mu M$ puromycin hydrochloride (Ameresco) in PBS for MPS assessment. Following pulse-labeling, cells were lysed using a cell lysis buffer as described above, homogenates were centrifuged at $500 \times g$ for $5 \mathrm{~min}$, and supernatants analyzed for total protein using a BCA assay (Pierce). Supernatants were then stored at $-20^{\circ} \mathrm{C}$ for Western blotting analyses as described below.

\section{Experiment 2: Effects of LAT1 Knockdown on MPS}

A separate batch of $\mathrm{C}_{2} \mathrm{C}_{12}$ myoblasts was seeded in 6 -well plates. Cells were grown to 40 to $50 \%$ confluency and then transfection growth medium (Opti MEM; ThermoFisher Scientific, Grand Island, NY) containing Lipofectamine 3000 (ThermoFisher Scientific) was added to myoblasts per the manufacturer's recommendations. Specifically, 6 wells were transfected with green fluorescent protein reporter plasmids (GeneCopoeia, Rockville, MD) containing either scrambled short-hair pinned RNA (shRNA; $250 \mathrm{ng} /$ well) or 3 different sequences of LAT1 shRNA (250 ng/well). Cells were differentiated $48 \mathrm{~h}$ after transfection, as described above. Cells were then allowed to differentiate for $7 \mathrm{~d}$ before imaging on an inverted fluorescent microscope (Eclipse Ci-L; Nikon Instruments Inc., Melville, NY) and digital capture (DS-QilMc; Nikon Instruments,
Inc.). We obtained $10 \times$ digital fluorometric fluorescein isothiocyanate (FITC)-filtered images to confirm transfection had occurred. Cells were then lysed with Ribozol (Ameresco) per the manufacturer's recommendations (http://www.amresco-inc.com/media. acux?path=/media/products/dfu/dfu-N580.pdf), and LAT1 mRNA knockdown was confirmed using realtime PCR as mentioned below. Specifically, all 3 LAT1 shRNA sequences caused a decrease in LAT1 mRNA compared with scrambled shRNA (relative expression units: scrambled shRNA $=1.00 \pm 0.54$; LAT1 shRNA sequence $1=0.42 \pm 0.06$; LAT1 shRNA sequence $2=$ $0.30 \pm 0.04 ;$ LAT1 shRNA sequence $3=0.25 \pm 0.05$ ).

Following confirmation of successful transfection and LAT1 mRNA knockdown, 2 separate batches of $\mathrm{C}_{2} \mathrm{C}_{12}$ myoblasts were seeded in 6 -well plates (1 batch for LAT1 mRNA analyses and 1 batch for protein analyses). Following LAT1 shRNA sequence 3 transfection and $7 \mathrm{~d}$ of differentiation as described above, cells from both batches were treated acutely with 1 of the following treatments for $6 \mathrm{~h}$ : (1) Leu-free DMEM (LAT1 control) only; (2) $750 \mu \mathrm{g} / \mathrm{mL}$ Leu (EMD Chemicals); or (3) $5 \mathrm{mg} / \mathrm{mL}$ hydrolyzed WP (Hilmar Ingredients). The Leu content of the Leu treatment dose was slightly higher than the Leu present in the WP treatment. Following treatments, and 30 min before cell lysis, cells for protein analyses were pulse-labeled with $1 \mu M$ puromycin hydrochloride (Ameresco) in PBS for MPS assessment. Following pulse-labeling, cells were lysed using a cell lysis buffer as described above, homogenates were centrifuged at $500 \times g$ for $5 \mathrm{~min}$, and supernatants analyzed for total protein using a BCA assay. Supernatants were then stored at $-20^{\circ} \mathrm{C}$ for Western blotting and phosphoproteomic analyses as described below. Following treatments, cells for LAT1 mRNA analyses were lysed with Ribozol (Ameresco) per the manufacturer's recommendations (http://www.amresco-inc.com/media.acux?path=/media/products/dfu/dfu-N580.pdf), and LAT1 mRNA knockdown was confirmed using real-time PCR as described below.

\section{Experiment 3}

Whey Protein-Derived Exosome Isolation. Whey protein-derived exosome isolation was performed as described previously (Yamada et al., 2012). Briefly, approximately $40 \mathrm{~g}$ of hydrolyzed WP (Hilmar Ingredients) was blended in $473 \mathrm{~mL}$ of sterile distilled water. Following suspension, WP samples were centrifuged at $5,000 \times g$ for $30 \mathrm{~min}$ at $4^{\circ} \mathrm{C}$ using a Heraeus Megafuge 16R centrifuge (ThermoFisher Scientific) to remove milk fat. Samples were then subjected to differential ultracentrifugation at $4^{\circ} \mathrm{C}$ in a WX Ultra 80 ultracen- 
trifuge (Fiberlite F37L-8 $\times 10$ rotor; ThermoFisher Scientific) whereby the protocol included $12,000 \times g$ for $1 \mathrm{~h}, 35,000 \times g$ for $1 \mathrm{~h}$, and $70,000 \times g$ for $3 \mathrm{~h}$. Following ultracentrifugation, supernatants were nanofiltered through $0.22-\mu \mathrm{m}$ filters (VWR, Radnor, PA) to yield WP serum. Immediately following nanofiltration, the WP serum was aliquoted into microcentrifuge tubes and subjected to exosome isolation using a reagentbased kit (Life Technologies). Briefly, $1 \mathrm{~mL}$ of serum was mixed with $500 \mu \mathrm{L}$ of exosome isolation reagent and incubated at $2-8^{\circ} \mathrm{C}$ overnight. The following day, samples were centrifuged at $100,000 \times g$ for $1 \mathrm{~h}$ at $4^{\circ} \mathrm{C}$. Supernatants were discarded, and the remaining pellet containing exosomes was retained and resuspended in $100 \mu \mathrm{L}$ of sterile $1 \times$ PBS. The resuspended pellets were used for the WP-EXO treatments described below.

Flow Cytometry, Western Blotting, and Transmission Electron Microscopy to Confirm WP$\boldsymbol{E X O}$ Isolation. Flow cytometry was employed using a BD Accuri flow cytometer (BD Biosciences, San Jose, $\mathrm{CA}$ ) to confirm the presence of WP-EXO following the isolation protocol. As described in a previous study (Lässer et al., 2012), isolated exosomes were labeled with an anti-bovine milk exosome specific tetraspanin, CD63 (Reinhardt et al., 2012) primary antibody (1:10; AbD Serotec, Atlanta, GA) and allowed to incubate for $1 \mathrm{~h}$. Following primary antibody incubation, isolated exosomes were washed and probed with a FITC-conjugated secondary antibody (1:50; AbD Serotec) and allowed to incubate for $30 \mathrm{~min}$. Following incubation, samples were washed and read in the flow cytometer.

Western blotting was also used to confirm the detection of WP-EXO. Briefly, WP-EXO resuspended pellet samples were diluted to a working protein concentration (1:20) using PBS and prepared for electrophoresis, which is described in detail below. Membranes were probed with an anti-bovine milk exosome specific tetraspanin, CD9 primary antibody (1:1,000; Abcam, Cambridge, GA; Reinhardt et al., 2012). Following overnight incubation, membranes were washed and probed with a horseradish peroxidase-conjugated antimouse IgG secondary antibody (1:2,000; Cell Signaling, Danvers, MA) and analyzed via chemiluminescent imaging as described below.

Finally, WP-EXO were subjected to transmission electron microscopy. Briefly, $5 \mu \mathrm{L}$ of resuspended WPEXO aliquots were placed on Formvar copper grids and allowed to sit for approximately $15 \mathrm{~min}$. Immediately following this, any remaining solution was wicked away. Subsequently, $5 \mu \mathrm{L}$ of $1 \%$ phosphotungstic acid, as used by previous research groups (Tracz et al., 1997; Smith and $\mathrm{Ma}, 2004$ ), was incorporated for negative staining. Following a brief incubation period, any remaining stain was wicked away and grids were analyzed using a Zeiss EM10 transmission electron microscope (Zeiss, Thornwood, NJ). Images of WP-EXO were captured at $100,000 \times$ magnification.

Treatments with WP-EXO. Following WP-EXO isolation and confirmation, a separate batch of $\mathrm{C}_{2} \mathrm{C}_{12}$ myoblasts were seeded in 6-well plates and differentiated as described above. For MPS experiments, myotubes were treated once for 6,12 , or $24 \mathrm{~h}$ with either differentiation medium and vehicle (PBS) or WP-EXO suspended in $100 \mu \mathrm{L}$ of PBS. Approximately $30 \mathrm{~min}$ before cell lysis, cells were pulse-labeled with $1 \mu M$ puromycin hydrochloride (Ameresco) in PBS for MPS assessment. Following pulse-labeling, cells were lysed using a cell lysis buffer as described above, homogenates were centrifuged at $500 \times g$ for $5 \mathrm{~min}$, and supernatants analyzed for total protein using a BCA assay. Supernatants were then stored at $-20^{\circ} \mathrm{C}$ for Western blotting and phosphoproteomic analyses as described below.

For assessment of WP-EXO on myotube hypertrophy, a separate batch of $\mathrm{C}_{2} \mathrm{C}_{12}$ myoblasts were seeded in 6-well plates and differentiated as described above. Myotubes were treated once for 24 or $48 \mathrm{~h}$ with either differentiation medium and vehicle (PBS) or WP-EXO suspended in $100 \mu \mathrm{L}$ of PBS. Following treatments, cells were fixed in $10 \%$ formalin and stored at $4^{\circ} \mathrm{C}$ until cell staining. Cell staining was performed per the modified methods of Veliça and Bunce (2011). Briefly, cells were removed from storage, thawed to room temperature, and incubated with $100 \%$ methanol for 5 min. Cells were then incubated with Jenner stain for 5 min (Electron Microscopy Sciences, Hatfield, PA) which was diluted at a 1:3 ratio with distilled water. Thereafter, cells were incubated with diluted Giemsa stain for 5 min (1:5 in distilled water; Electron Microscopy Sciences). Cells were rinsed with distilled water, wells were bright field imaged with a $10 \times$ objective (Eclipse Ci-L; Nikon Instruments, Inc.), and digital images were analyzed for fiber diameter using ImageJ (National Institutes of Health, Bethesda, MD). Specifically, fiber diameters of approximately 30 myotubes per well (180 myotubes per condition) were quantified.

\section{Directed Intracellular Phosphoproteomics}

A phosphoproteomic array (PathScan Intracellular Signaling Array Kit, Chemiluminescent Readout; Cell Signaling) containing glass slides spotted with antibodies was used to detect phosphorylated (p) proteins predominantly belonging to various intracellular signaling networks in experiments 2 and 3. Assayed targets included p-ERK (Thr202/Tyr204), p-Stat 1 (Tyr701), p-Stat 3 (Tyr705), p-Akt (Thr308), p-Akt (Ser473), 
p-rpS6 (Ser235/236), p-AMPK $\alpha$ (Thr172), p-PRAS40 (Thr246), p-mTOR (Ser2481), p-HSP27 (Ser78), pGSK- $3 \beta$ (Ser9), p-p70s6k (Thr389), p53 (Ser15), p-38 (Thr180/Tyr182), p-SAPK/JNK (Thr183/Tyr185), p-PARP (Asp214), p-Caspase-3 (Asp175), and p-BAD (Ser112). However, we specifically analyzed p-Akt (Ser473), p-AMPKa (Thr172), p-mTOR (Ser2481), p-p70s6k (Thr389), and p-rps6 (Ser235/ 236). Details of the procedure are described in detail elsewhere (McGinnis et al., 2015). Briefly, cell lysates were diluted to $1.0 \mu \mathrm{g} / \mu \mathrm{L}$ using the cell lysis buffer provided with the kit. Slides were developed using an enhanced chemiluminescent reagent provided with the kit, and spot densitometry was performed using a gel documentation system software (UVP LLC, Upland, CA). The calculation of each phosphorylated target was as follows: (density value of the target - negative control)/summation of all density values for the sample.

\section{Western Blotting Methods and Analysis}

The surface sensing of translation method was used to analyze MPS levels for all experiments as previously described by our laboratory (Roberts et al., 2015). Briefly, lysates were prepared with $4 \times$ Laemmli buffer and subjected to gel electrophoresis using $12 \%$ SDS-polyacrylamide pre-casted gels (BioRad, Hercules, CA). Proteins were transferred to polyvinylidene difluoride membranes (BioRad) and Ponceau stained to ensure equal loading of proteins. Bright field images of Ponceau stains were captured to normalize Western blotting results. Following Ponceau staining, membranes were blocked for $1 \mathrm{~h}$ at room temperature with $5 \%$ nonfat milk powder. Mouse anti-puromycin (1:5,000; Millipore, Billerica, MA) was then incubated with membranes overnight at $4^{\circ} \mathrm{C}$ in $5 \%$ bovine serum albumin. The following day, membranes were incubated with anti-mouse IgG secondary antibodies (1:2,000; Cell Signaling) at room temperature for $1 \mathrm{~h}$. Membranes were then developed using an enhanced chemiluminescent reagent (GE Healthcare Bio-Sciences, Pittsburgh, PA), and band densitometry was performed through the use of a gel documentation system and associated software (UVP). Specifically, whole-lane densitometry using rectangular regions of interest was performed to determine total protein synthesis, and these values were divided by the respective Ponceau densities to obtain relative density units for each sample.

Similar methods were used to assess LAT1, phosphatase and tensin homolog (PTEN), and eIF4A protein levels. Specifically, after Ponceau staining and membrane bright field imaging membranes were blocked for $1 \mathrm{~h}$ at room temperature with $5 \%$ nonfat milk powder.
Goat anti-LAT1 (1:1,000; Sigma, St. Louis, MO), rabbit anti-PTEN (1:1,000; Cell Signaling), and rabbit anti-eIF4A (1:1,000; Cell Signaling) were incubated with membranes overnight at $4^{\circ} \mathrm{C}$ in $5 \%$ bovine serum albumin. The following day, membranes were incubated with either horseradish peroxidase-conjugated anti-goat IgG (1:5,000; Santa Cruz Biotechnology, Dallas, TX) or anti-rabbit IgG secondary antibodies (1:2,000; Cell Signaling) at room temperature for $1 \mathrm{~h}$. Membranes were then developed using an enhanced chemiluminescent reagent (Amersham), and band densitometry was performed using a gel documentation system as described above, whereby the densities of each target of interest were divided by Ponceau densities.

\section{RNA Isolation and Real-Time PCR}

When applicable, RNA was isolated from myotubes grown on 6-well plates using Ribozol (Ameresco) per the manufacturer's recommendations (http://www. amresco-inc.com/media.acux?path=/media/products/ dfu/dfu-N580.pdf). Then, $500 \mathrm{ng}$ to $1 \mu \mathrm{g}$ of cDNA was synthesized using a cDNA synthesis kit (Quanta Biosciences, Gaithersburg, MD) per the manufacturer's recommendations (http://www.quantabio. com/media/wysiwyg/pdfs/95047_qScript_cDNA_Synthesis_Kit_PPS_150319.pdf). Real-time PCR was performed using mRNA-specific primers [experiment 2, SLC7A5/LAT1: forward GAGACCCTAGAGATGGAACCC, reverse TCACATCACACTGGTGACAGAG; experiment 3, eukaryotic initiation factor 4A (eIF4A): forward GATGTCCAGGTCTCCTT, reverse CCTTACGACCAAACCGACCA; experiment 3, PTEN: forward AACGGGCTTGATGTCTCAGG, reverse AACCTCCCATGTGCTGATGG; experiment 3, MyoD: forward CCTGCCCTCCACATCCTTTT, reverse GAAGGGGGAGAGTGGGGTAT; experiment 3, myogenin: forward GAGACATGAGTGCCCTGACC, reverse CAGAGGCTTTGGAACCGGAT; experiment 3, myosin heavy chain IIa (MyHCIIa): forward ATCTCAGGAGCACCCATCCT, reverse GGGAAGCCCTTTCTGCAGAT; experiments 2 and 3, Beta-glucoronidase (housekeeping gene): forward TCAGCTCTGTGACCGATACG, reverse GCCACAGACCACATCACAAC] and SYBR green chemistry (Quanta Biosciences). Fold-change values from the control condition of applicable experiments were performed using the Livak method (i.e., $2^{-\Delta \Delta \mathrm{CT}}$ assuming $100 \%$ primer binding efficiency), where $2^{-\Delta \mathrm{CT}}$ $=$ (housekeeping gene) $\mathrm{CT}-$ gene of interest $\mathrm{CT}$ and $2^{-\Delta \Delta \mathrm{CT}}$ (or fold-change) $=\left[2^{-\Delta \mathrm{CT}}\right.$ value of experimental condition $/ 2^{-\Delta \mathrm{CT}}$ average of control condition]. Of note, beta2-microglobulin was used as a housekeeping 
gene because it remained stable across all treatments. Primer efficiency curves for all genes were generated, and efficiencies ranged between 90 and $110 \%$. Melt curve analyses were performed to confirm that $1 \mathrm{PCR}$ product was obtained.

\section{Global Bovine miRNA Microarray Analysis of WP-EXO and WP-EXO-Treated Myotubes}

Global bovine miRNA were analyzed from WP-EXO to observe bovine miRNA that were enriched from isolated exosomes. Moreover, to determine if WP-EXO treatments "transferred" bovine miRNA to myotubes, global bovine miRNA expression profiles were analyzed from a subset of control and $24 \mathrm{~h}$ WP-EXO-treated myotubes in experiment 3 ( $\mathrm{n}=3$ for each treatment). Specifically, $2 \mu \mathrm{g}$ of total RNA from each sample was sent to a commercial laboratory and subjected to miRBase 21 Bos taurus (bta) specific miRNA probing (LC Sciences LLC, Houston, TX) as described in a previous study (Liu et al., 2015).

\section{Statistics}

All data are presented as mean \pm standard error. For all data, statistics were performed between treatments using an ANOVA with LSD post hoc comparisons or independent 2-tailed $t$-tests when applicable. All statistics were performed using SPSS version 22.0 (IBM Corp., Armonk, NY) and significance was set at $P<$ 0.05 , although $P$-values $<0.01$ and $<0.001$ were also denoted throughout.

\section{RESULTS}

\section{WP Stimulates Greater Increases in Myotube MPS Compared with EAA and Leu}

Compared with control myotubes, Leu, EAA, and WP equally stimulated MPS $3 \mathrm{~h}$ after treatment $(\sim 20-30 \%)$, although we observed no significant differences between groups (ANOVA, $P=0.13$ ). However, MPS levels with WP were $46 \%$ greater than control $(P$ $=0.001), 24 \%$ greater than Leu $(P<0.05)$, and $29 \%$ a

\section{LEU vs. EAA vs. WP effects on myotube MPS}

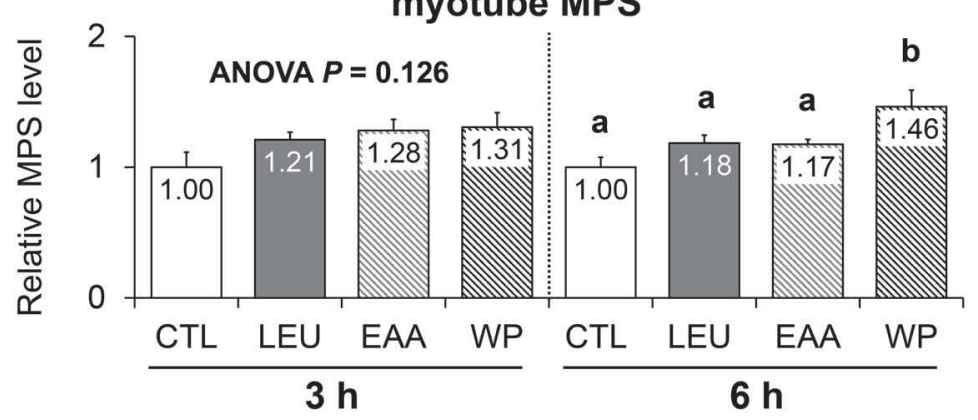

b

\section{Low-, mid-, high LEU vs. WP effects on} myotube MPS

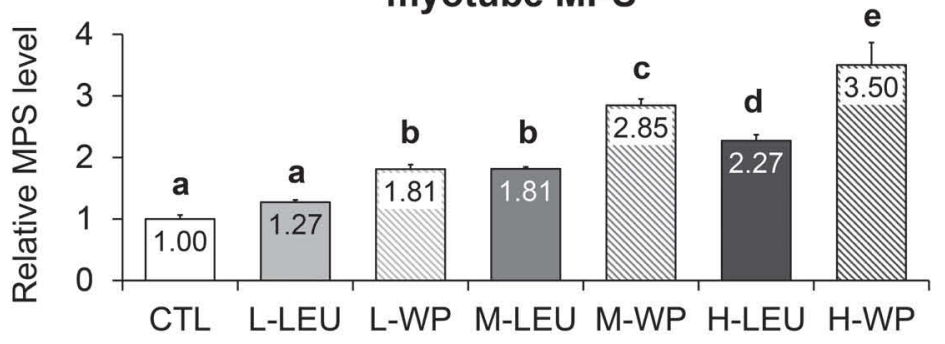

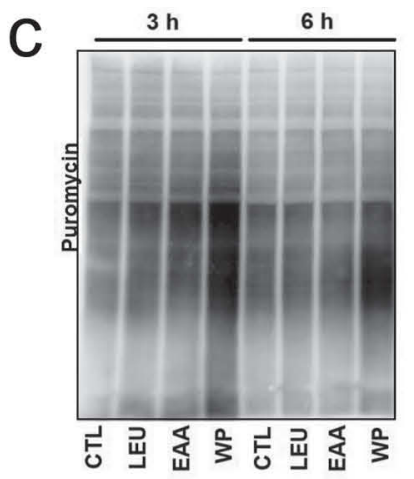
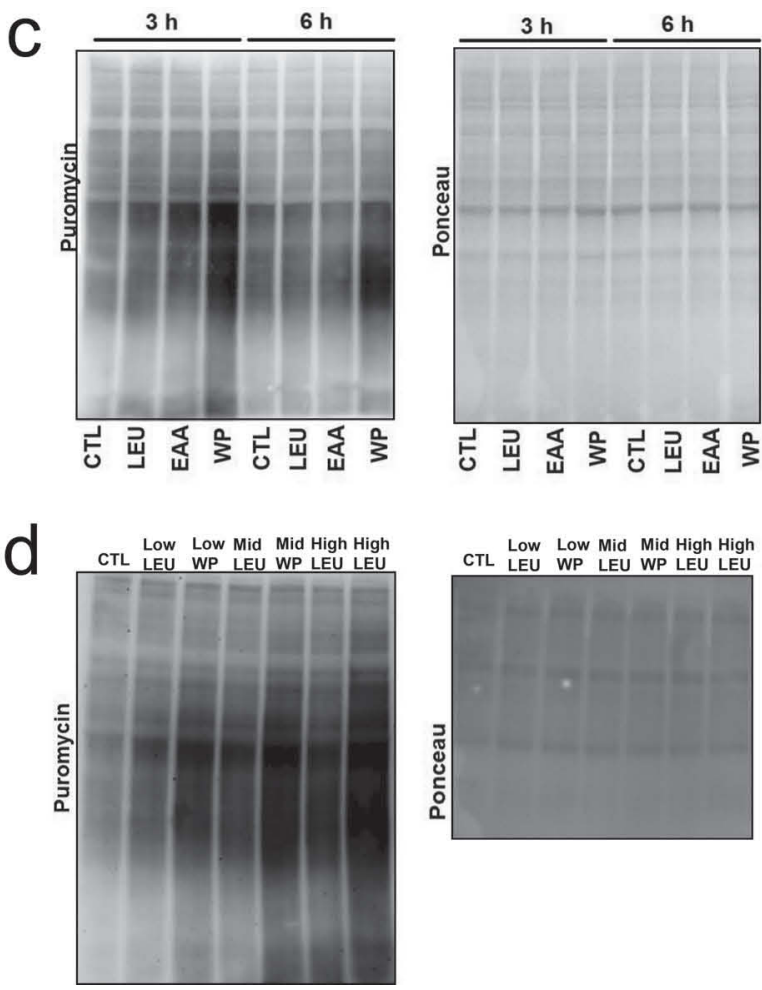

Figure 1. Effects of leucine (Leu), EAA, or whey protein (WP) treatments on myotube muscle protein synthesis (MPS). (a) Myotube MPS levels with 3-h and 6-h control (CTL), Leu, EAA, or WP treatments; (b) myotube MPS levels with 6-h control or low (L)-, medium (M)-, and high (H)-dose Leu or WP treatments; (c) representative surface sensing of translation (SUnSET) Western blot images for panel (a); (d) representative SUnSET Western blot images for panel (b). Data are presented as mean $\pm \mathrm{SE}$ ( $\mathrm{n}=6$ per bar for all groups). One-way ANOVA with a Fisher's least significant difference (LSD) post hoc test was performed; significant between-treatment differences are represented by different letters $(\mathrm{a}-\mathrm{e} ; P<0.05)$. 
greater than EAA $(P<0.05)$ at $6 \mathrm{~h}$ posttreatment (Figure 1a).

\section{Leu Dose Contributes Minimally to the Myotube MPS Response to WP}

Given that the WP myotube MPS response was more robust at $6 \mathrm{~h}$ than at $3 \mathrm{~h}$, the WP and Leu doseresponse experiments were all performed at $6 \mathrm{~h}$ (Figure 1b). Compared with control myotubes, we observed no increase in myotube MPS after low-dose Leu treatment $(P=0.30)$. However, low-dose WP increased myotube MPS by 81 and $54 \%$ compared with control and lowdose Leu, respectively $(P<0.001)$. Compared with control myotubes, medium-dose Leu increased myotube MPS by $81 \%(P<0.001)$, similar to low-dose WP, and medium-dose WP treatment increased myotube MPS by $185 \%(P<0.001)$ and $104 \%$ compared with low-dose WP and medium-dose Leu $(P<0.001)$. Compared with control myotubes, high-dose Leu increased myotube MPS by $127 \%(P<0.001)$ and high-dose WP increased myotube MPS by $250 \%(P<0.001)$. Myotube MPS was $123 \%$ greater in high-dose WP compared with high-dose Leu $(P<0.001)$.

\section{Anabolic Effects of WP Persist Beyond LAT1 Knockdown}

Transfection of LAT1 shRNA revealed a robust response, as seen with green fluorescent protein-positive myotubes (Figure 2a). Levels of LAT1 mRNA were $41 \%$ lower in LAT1-shRNA-transfected myotubes than in control myotubes transfected with scrambled shRNA $(P<0.001 ;$ Figure $2 \mathrm{~b})$, and LAT1 protein was $20 \%$ lower in LAT1-shRNA-transfected myotubes than in control myotubes transfected with scrambled shRNA $(P<0.05$; Figure 2b). Compared with LAT1-shRNAtransfected control myotubes, LAT1-shRNA-transfected myotubes treated with medium-dose Leu for $6 \mathrm{~h}$ decreased p-Akt (Ser473) by 35\% $(P<0.01$; Figure 2c) and p-AMPK $\alpha$ (Thr172) by $25 \%(P<0.01$; Figure $2 \mathrm{~d})$. Likewise, LAT1-shRNA-transfected myotubes treated with medium-dose WP for 6 h decreased p-Akt (Ser473) by $33 \%(P<0.01$; Figure $2 \mathrm{c})$ and p-AMPK $\alpha$ (Thr172) by $33 \%(P<0.001$; Figure 2 d) compared with LAT1shRNA-transfected control myotubes. In addition, MPS in LAT1-shRNA-transfected myotubes treated with medium-dose WP for $6 \mathrm{~h}$ was $18 \%$ higher than control LAT1-transfected myotubes and 19\% higher than Leu LAT1-transfected myotubes $(P<0.05$; Figure $2 \mathrm{~h})$. We observed no treatment effects for p-mTOR (Ser2448), p70s6k (Thr389), or p-rps6 (Ser235/236) compared with controls $(P>0.05)$. Notwithstanding, myotube MPS in WP-treated LAT1-shRNA-transfected myotubes was increased $18 \%$ compared with control LAT1-shRNAtransfected myotubes $(P<0.05)$ and $19 \%$ compared with Leu-treated LAT1-shRNA-transfected myotubes $(P<0.05 ;$ Figure 2i).

\section{Confirmation of WP-EXO Using Various Biochemical and Imaging Techniques}

Flow cytometry confirmed that WP-EXO isolates contained bovine-specific exosomes due to the presence of small CD63 labeled particles (Figure 3a). We further confirmed the isolation and presence of WP-EXO through the detection of CD9 via Western blotting (Figure 3b). Finally, transmission electron microscopy visually confirmed the presence of exosome-like particles from WP-EXO isolates (Figure 3c).

\section{Effects of WP-EXO on Myotube Anabolic Signaling, MPS, and Myotube Size}

Given that (1) WP was more anabolic than EAA and Leu; (2) WP was more anabolic at low, medium, and high doses versus comparable doses of Leu; and (3) the anabolic effects of WP persisted beyond LAT1mediated transport as detailed above, we next examined whether WP-EXO could be responsible for the additional anabolic effects observed with WP treatments versus single amino acids.

We first determined whether myotubes treated with WP-EXO affected phospho-signaling at $6 \mathrm{~h}$ or $24 \mathrm{~h}$ (Figure 4). Following 6-h treatments, p-Akt (Ser473) had a tendency $(P=0.066)$ to be diminished in the WPEXO treatments by $30 \%$, and we observed no difference between WP-EXO and control for (Ser473) after $24 \mathrm{~h}$ $(P>0.05)$ (Figure 4a). We observed that p-AMPK $\alpha$ (Thr172) was significantly $(+43 \% ; P<0.01)$ increased after WP-EXO compared with control treatments at the $24 \mathrm{~h}$ time point (Figure 4b). However, p-mTOR (Ser2448), p-p70s6k1 (Thr389), and rpS6 (Ser235/236) were not affected by WP-EXO treatments (Figure 4ce).

Given that WP-EXO did not appreciably affect the mTORC1 downstream signaling substrates as described above, we determined whether WP-EXO affected PTEN and eIF4A mRNA and protein expression, given that these are putative anabolic targets of bovinederived exosomal miRNA that are explicit to WP-EXO (Melnik et al., 2013; Melnik, 2015). Phosphatase and tensin homolog is a negative regulator of mTOR signaling, and eIF4A is a eukaryotic initiation factor required for mRNA to associate with the $40 \mathrm{~S}$ subunit during translation initiation. Interestingly, PTEN mRNA expression was decreased by 27 and $29 \%$ at 12 and $24 \mathrm{~h}$ $(P<0.05$ and $P<0.01$, respectively) in WP-EXO- 

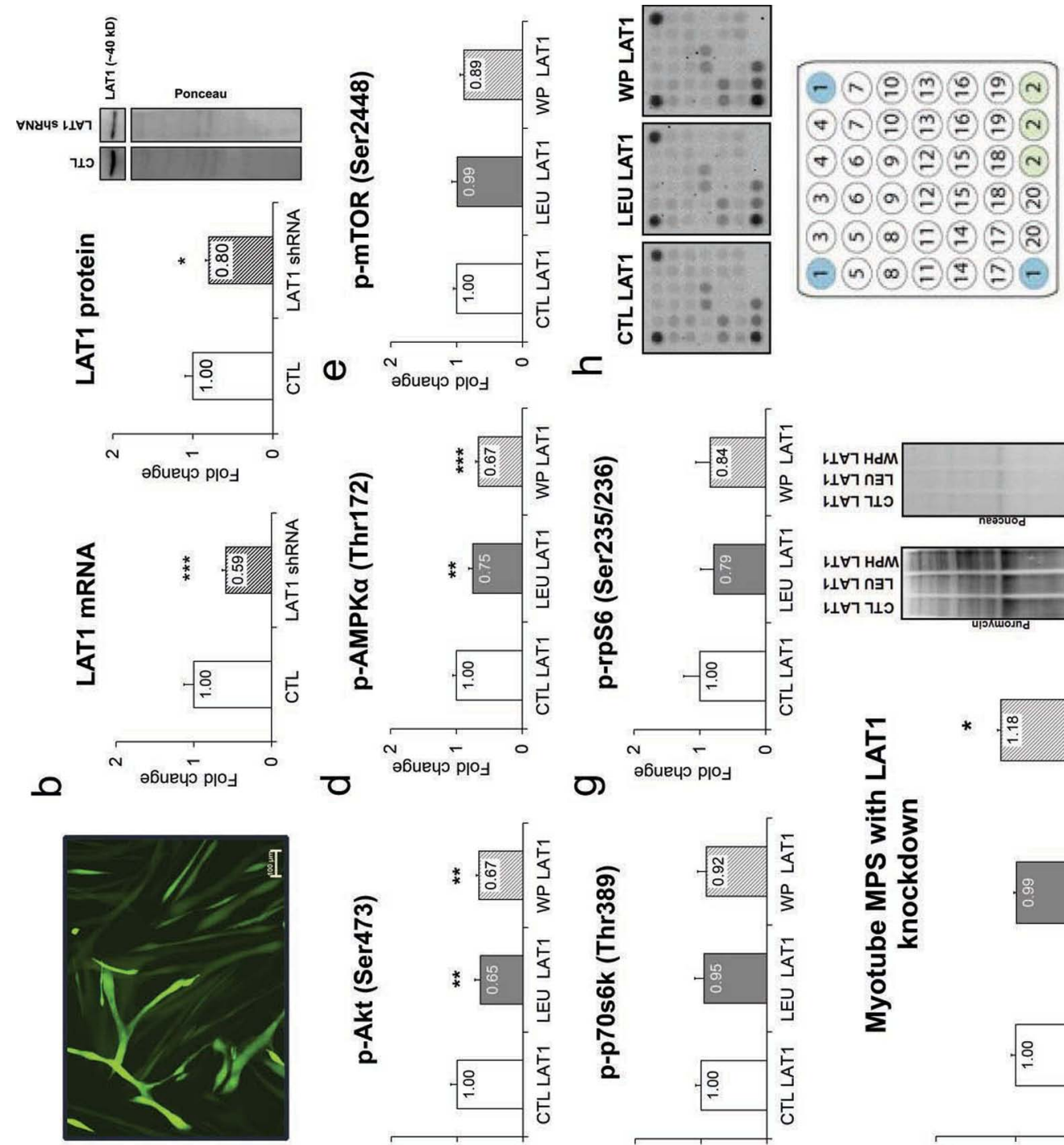

চ

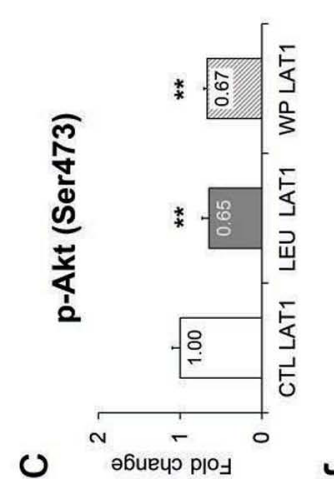

๑)
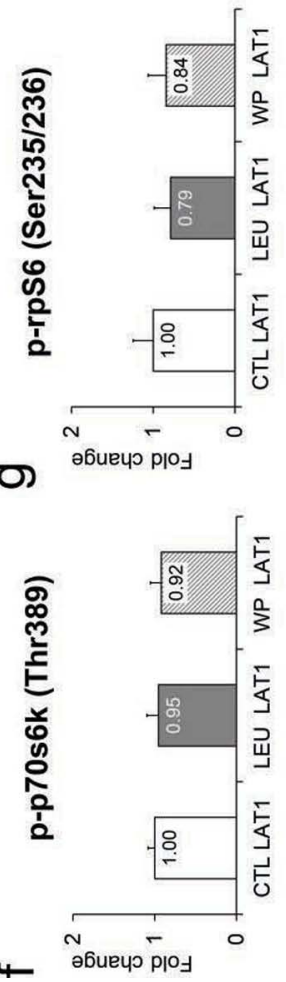

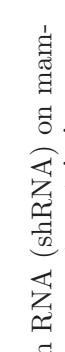

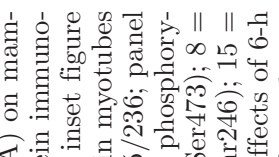

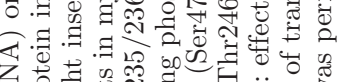

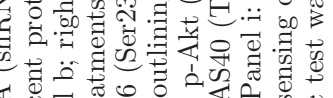

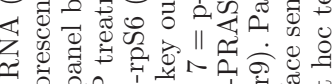

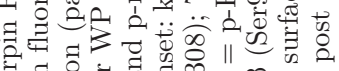

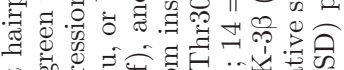

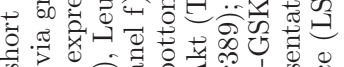

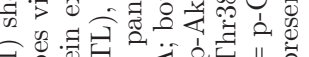

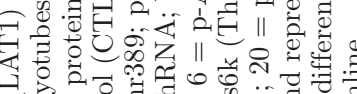

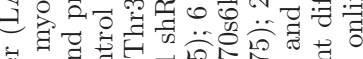

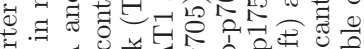

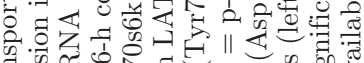

की

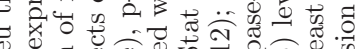

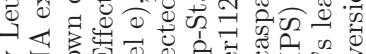

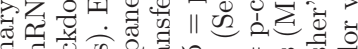

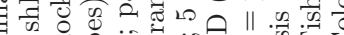

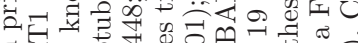

견

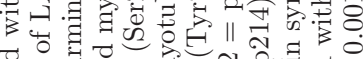

ช

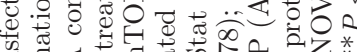

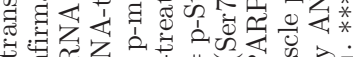

Ð

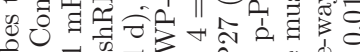

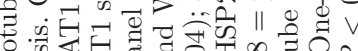

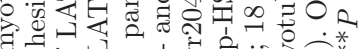
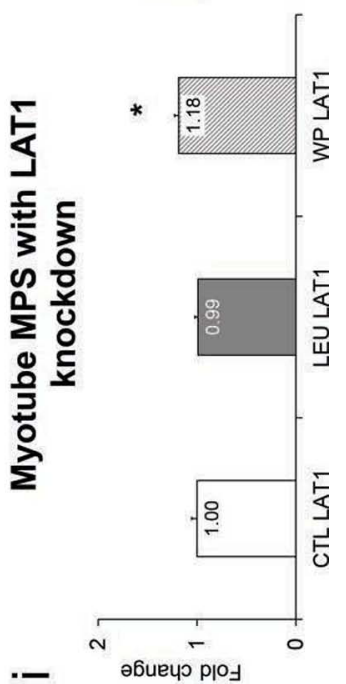

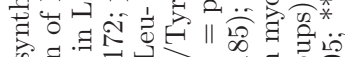

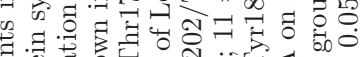

讨

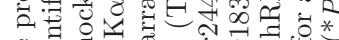

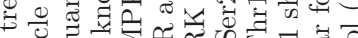

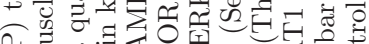

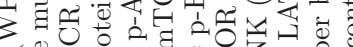

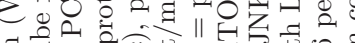

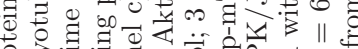

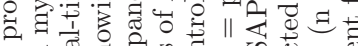
هั

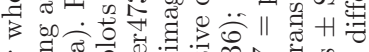
ธั 离.

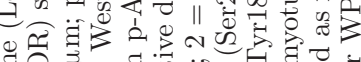

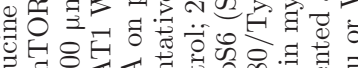
区

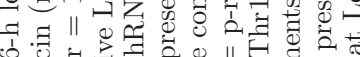

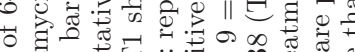

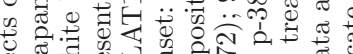

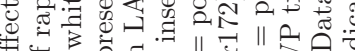

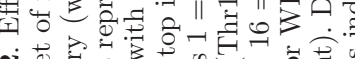

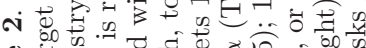

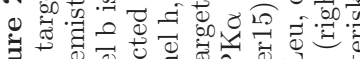
o 0 过

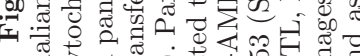


treated myotubes (Figure 5a) compared with controls, although we observed no treatment effects regarding PTEN protein expression (Figure 5b). We observed a trend for a $54 \%$ increase in eIF4A mRNA expression $6 \mathrm{~h}$ after WP-EXO treatment compared with controls but this finding failed to reach significance $(P=0.052$; Figure 5c). In contrast, eIF4A mRNA expression was $32 \%$ lower than control-treated myotubes after $24 \mathrm{~h}(P$ $<0.01$; Figure 5c). As well, eIF4A protein expression increased in WP-EXO-treated myotubes by $24 \%$ with the $12 \mathrm{~h}$ treatment; this tended to be greater than control-treated myotubes $(P=0.09$; Figure $5 \mathrm{~d})$ and was $17 \%$ higher after $24 \mathrm{~h}(P<0.05$; Figure $5 \mathrm{~d})$. Notably, for the PCR data presented above, critical threshold values for $\beta$-glucuronidase (housekeeping gene) were not statistically different at $6 \mathrm{~h}$ (control $27.62 \pm 0.48$, WP-EXO 27.68 $\pm 0.58 ; P=0.94), 12 \mathrm{~h}$ (control 28.41 \pm 0.88 , WP-EXO $27.31 \pm 0.32 ; P=0.27)$ or $24 \mathrm{~h}$ (control $27.57 \pm 0.38$, WP-EXO $27.03 \pm 0.26 ; P=0.26$ ).

Although WP-EXO did not increase mTORC1 signaling, the WP-EXO-induced increase in eIF4A led us to examine the effects of WP-EXO on MPS at $6 \mathrm{~h}, 12$ $\mathrm{h}$, and $24 \mathrm{~h}$. At $6 \mathrm{~h}$, WP-EXO treatments exhibited a nonsignificant $20 \%$ increase in myotube MPS compared with controls $(P=0.09$; Figure 5e). Interestingly, myotube MPS was $18 \%$ higher in the WP-EXO-treated myotubes after $12 \mathrm{~h}(P<0.05$; Figure 5e); this anabolic response persisted after $24 \mathrm{~h}$ and resulted in a $45 \%$ increase in MPS compared with controls $(P<0.05$; Figure 5e).

Beyond mTORC1 signaling and MPS, we were also interested in examining how WP-EXO treatments affected the mRNA expression of key myogenic regulatory factors involved with early- and late-stage differentiation (MyoD and myogenin, respectively; Figure 6a and $6 \mathrm{~b}$ ), as well as the mRNA expression of MyHCIIa (a mature myotube gene; Figure 6c). After 6 h treatments, WP-EXO did not affect the expression of any of the aforementioned mRNA. However, WP-EXO treatments significantly downregulated MyoD mRNA levels at 12 $\mathrm{h}(P<0.05)$, and this downregulation persisted at 24 h $(P<0.01)$. Similarly, WP-EXO treatments tended to downregulate myogenin mRNA at $12 \mathrm{~h}(P=0.08)$, and downregulation was significant at $24 \mathrm{~h}(P<0.01)$. Together, these data suggest that WP-EXO treatments do not enhance the differentiation state of the remaining myoblasts in culture. Notably, critical threshold values for $\beta$-glucuronidase (housekeeping gene) were stable and were the same values as used for mRNA data in Figure 5.

The effects of 24 and $48 \mathrm{~h}$ WP-EXO treatments on myotube diameters are presented in Figure 7. Remark- a

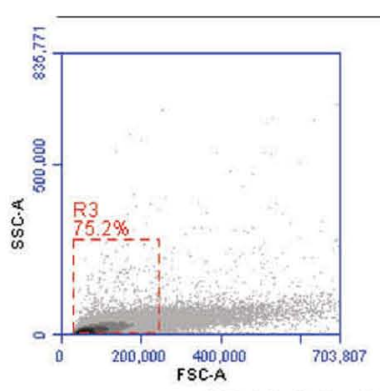

Unlabeled WP-EXO

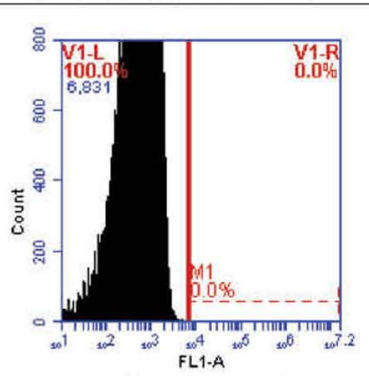

CD63-labelled WP-
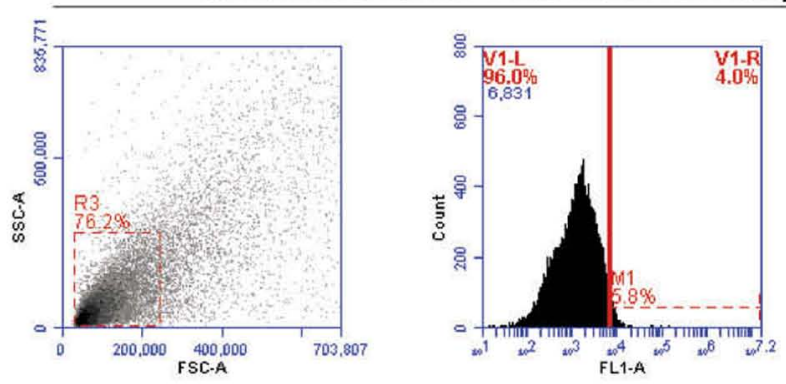

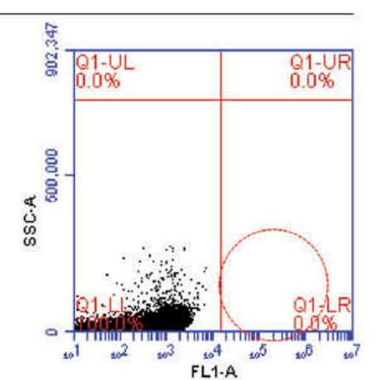

gated 2' antibody

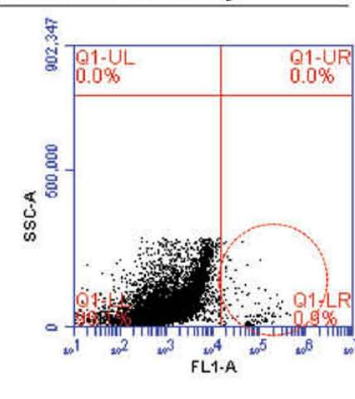

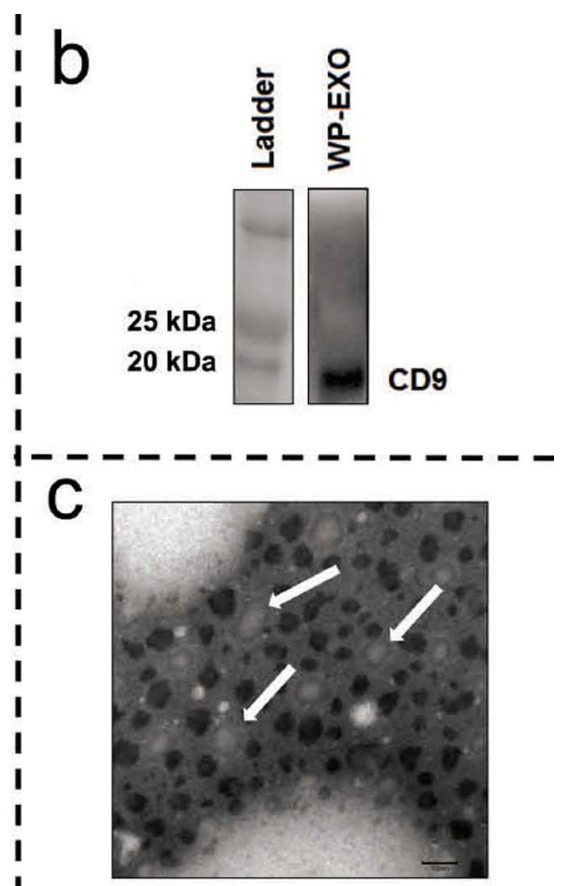

Figure 3. Confirmation of successful isolation of whey protein-derived exosomes (WP-EXO): (a) using flow cytometry with surface marker CD63 for detection (FITC = fluorescein isothiocyanate); (b) using Western blotting using surface marker CD9 for detection, with (c) visual confirmation of WP-EXO isolation via transmission electron microscopy $(100,000 \times)$; white arrows pinpoint WP-EXO (black bar $=100 \mathrm{~nm})$. Color version available online. 

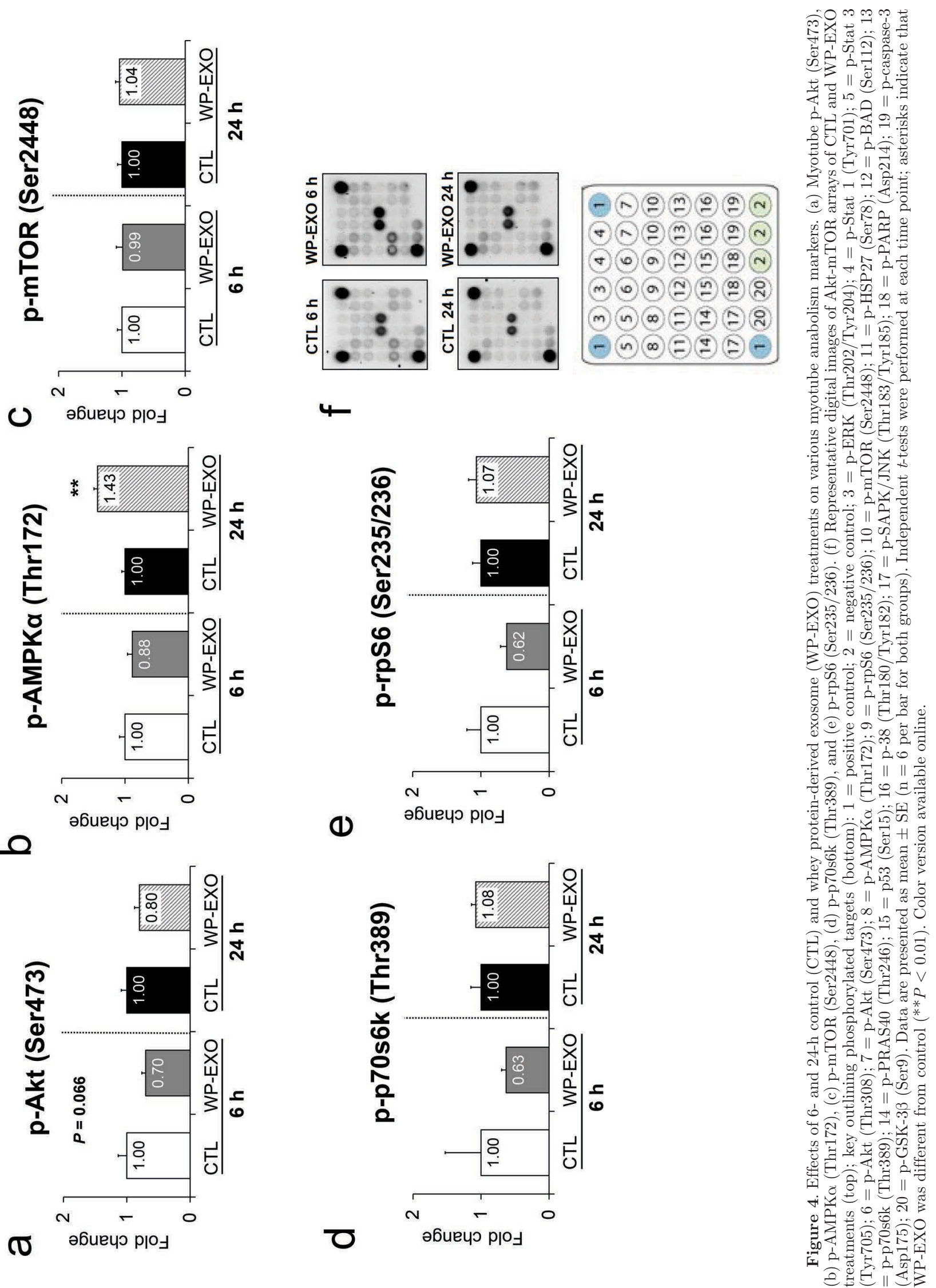

(1)
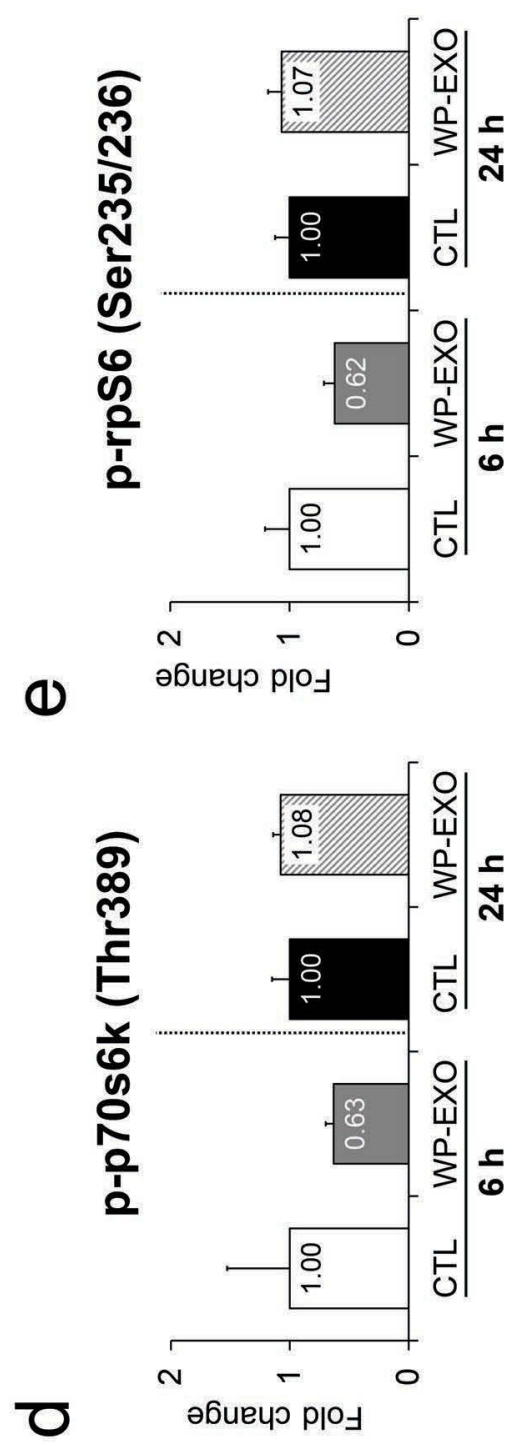

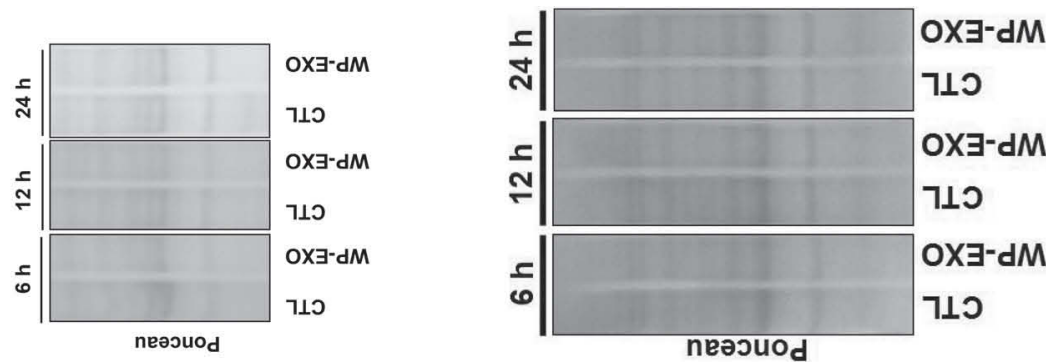

运学泀官

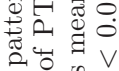

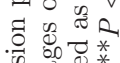

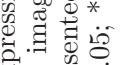

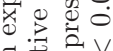

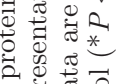
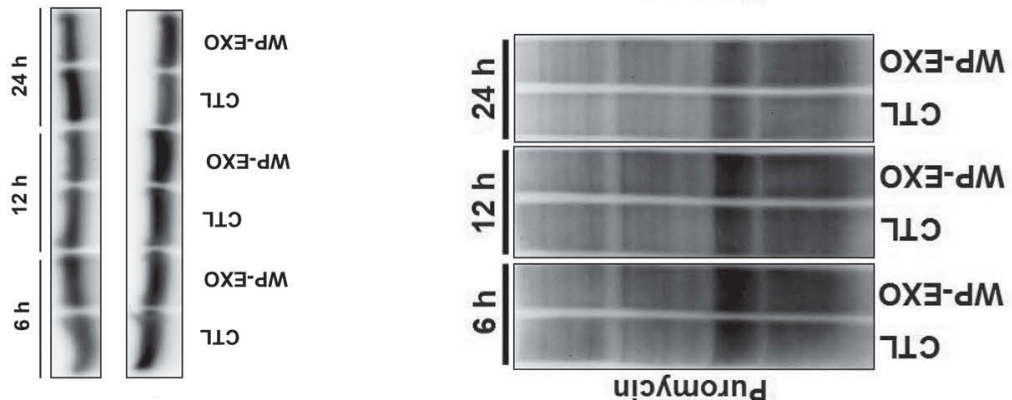

สิ का

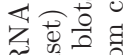

邹.

造施

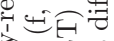

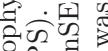

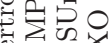

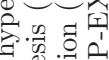

趈 壳

o 감 $\frac{\pi}{62}$

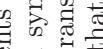

구웡

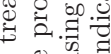

4
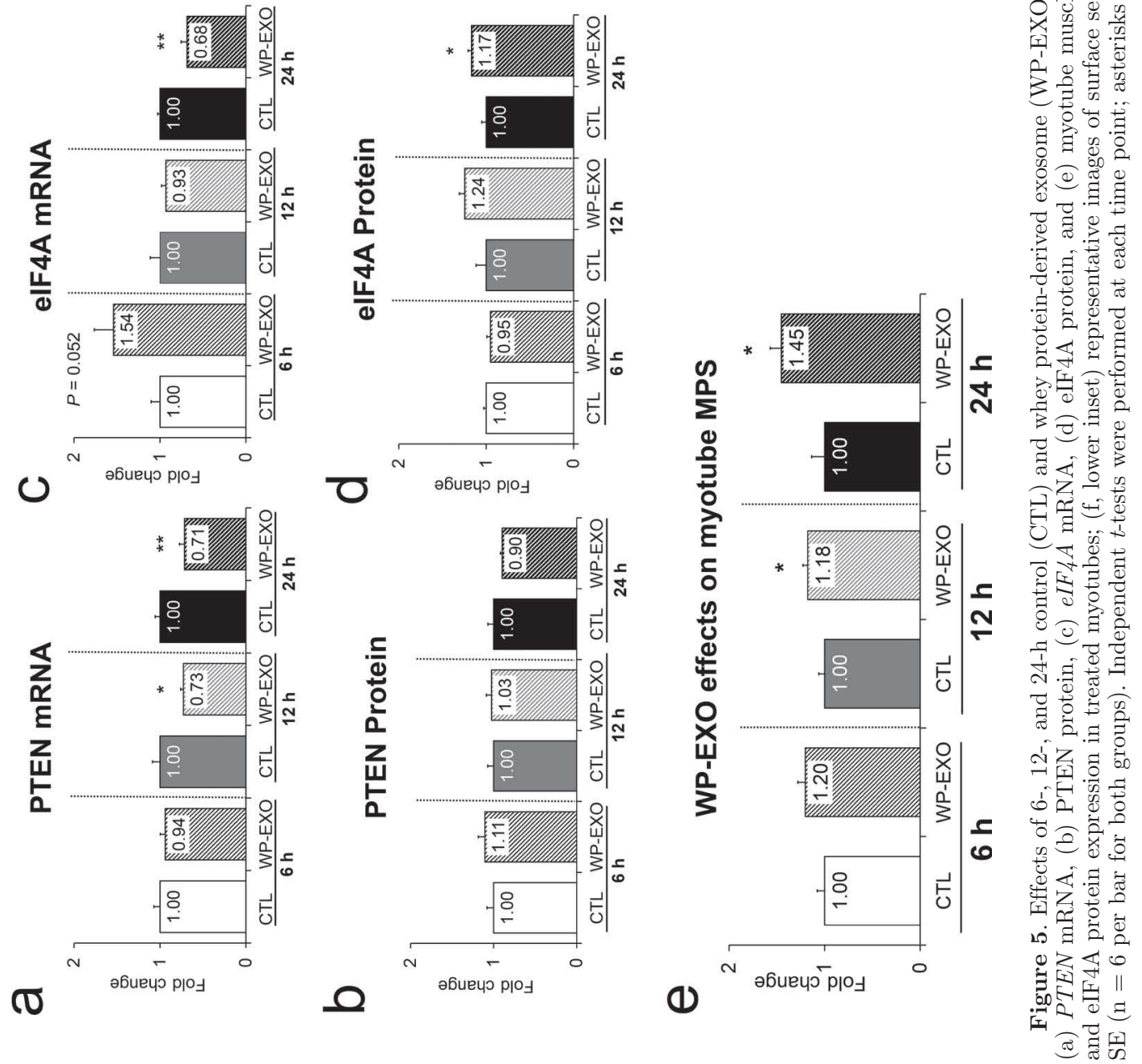
ably, both $24 \mathrm{~h}(P<0.001)$ and $48 \mathrm{~h}$ treatments $(P<$ $0.001)$ significantly increased myotube diameters compared with PBS-treated (control) myotubes.

\section{Bovine MicroRNAs Elevated in the RNA Pool of WP-EXO-Treated Myotubes}

Global bovine miRNA microarray analysis revealed that bta miR-149-3p, a negative regulator of Akt1 (Lin et al., 2010), was $44 \%$ more highly enriched in WP-EXO than in controls after $24 \mathrm{~h}(P=0.021)$. Similarly, bta miR-214, an inhibitor of PTEN (Yang et al., 2013), was $35 \%$ more highly enriched in WP-EXO than in control myotubes after $24 \mathrm{~h}(P=0.049)$. Other bovine-specific miRNA that were differentially present in WP-EXO versus control myotubes included bta miR-30b $(P=$ 0.006), bta miR-16b $(P=0.044)$, bta-let7i (tendency at $P=0.064$ ), and bta miR-6520 (tendency at $P=0.068$ ). Other miRNA of interest, according to recent review articles (Melnik et al., 2013; Melnik, 2015), included bta miR-21 [a suppressor of PTEN expression (Han et al., 2012) and PDCD4 (Asangani et al., 2008)] and miR29b (a suppressor of the branched- chain keto acid dehydrogenase complex); however, these targets were equally present $(P=0.46$ and $P=0.11$, respectively) in WP-EXO and control myotubes after $24 \mathrm{~h}$. A summary of these findings, as well as bovine miRNA detected in the WP-EXO isolate itself, are presented in Table 2.

\section{DISCUSSION}

To our knowledge, this is the first study to demonstrate that hydrolyzed WP contains bioactive exosomes that enhance protein synthesis and anabolism in skeletal muscle cells independent of amino acids in vitro. Moreover, although WP-EXO did not affect mTOR signaling 6 to $24 \mathrm{~h}$ after WP-EXO treatments in the current study, their effects on myotube anabolism may be derived from either earlier increases in mTORC1 signaling, which we did not capture with our experimental design (i.e., 1-3 h), or the increased expression of eukaryotic initiation factors, as we observed in the present study.

Compared with other whole food sources, WP contains a high Leu content (Hulmi et al., 2010), and the consumption of WP has been shown to increase postprandial plasma Leu levels, which is associated with increases in MPS (Tang et al., 2009). However, the current body of scientific literature is inconsistent regarding the anabolic effects of "high-quality" protein sources that contain a high content of EAA (i.e., milk protein versus isolated WP versus isolated soy protein versus isolated casein protein). Phillips et al. (2009) have used meta-analysis to demonstrate that WP is more anabolic than soy protein supplementation in human clinical trials, both protein sources being rich in Leu. Indeed, the authors attributed the superior anabolic response of WP to the more rapid digestibility and increased pharmacokinetic profile of postprandial leucine. However,

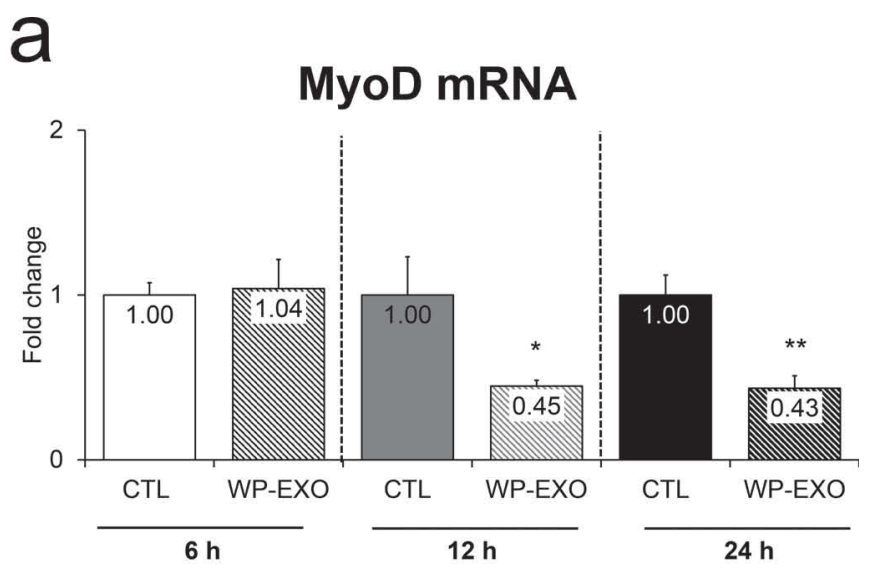

$b$

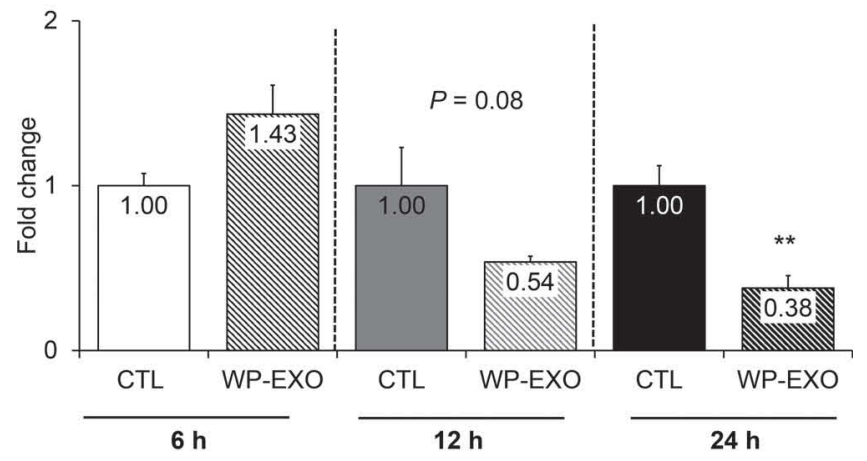

C
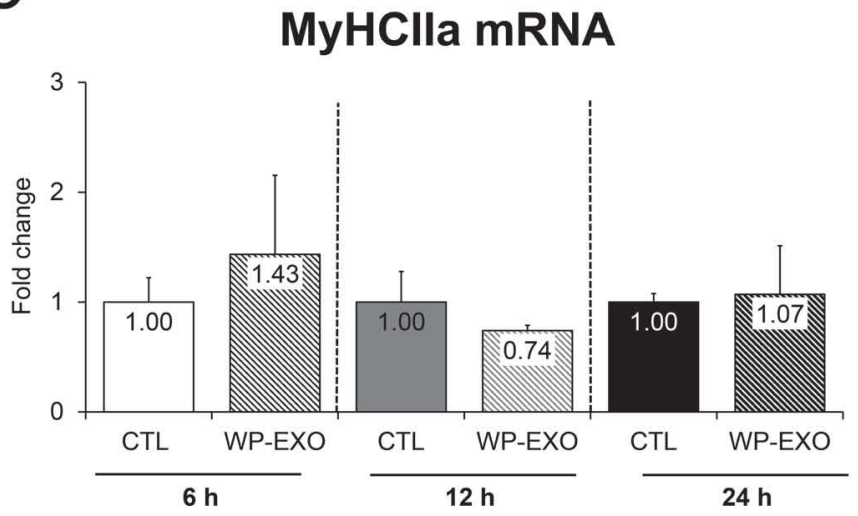

Figure 6. Effects of 6-, 12-, and 24-h control (CTL) and whey protein-derived exosome (WP-EXO) treatments on mRNA expression patterns related to differentiation: (a) MyoD mRNA, (b) myogenin mRNA, and (c) MyHCIIa mRNA. Data are presented as means $\pm \mathrm{SE}$ ( $\mathrm{n}=6$ per bar for all groups). Independent $t$-tests were performed at each time point; asterisks indicate that WP-EXO was different from control $\left({ }^{*} P<0.05 ; * * P<0.01\right)$. 
additional bioactives in bovine-derived WP versus soy protein may exist that account for the additional hypertrophic effects of WP. In support of this hypothesis, Hartman et al. (2007) reported that daily milk supplementation was superior to isonitrogenous soy protein supplementation in terms of increasing muscle mass in resistance-trained participants. This study is fascinating given that the amount of milk consumed, although it proved to be more anabolic than soy, provided only $\sim 17 \mathrm{~g}$ of milk protein per day (roughly $3.5 \mathrm{~g}$ of WP and $14.5 \mathrm{~g}$ casein). High-dose casein protein supplementation $(1.5 \mathrm{~g} / \mathrm{kg} / \mathrm{d})$ has proven to be futile in promoting

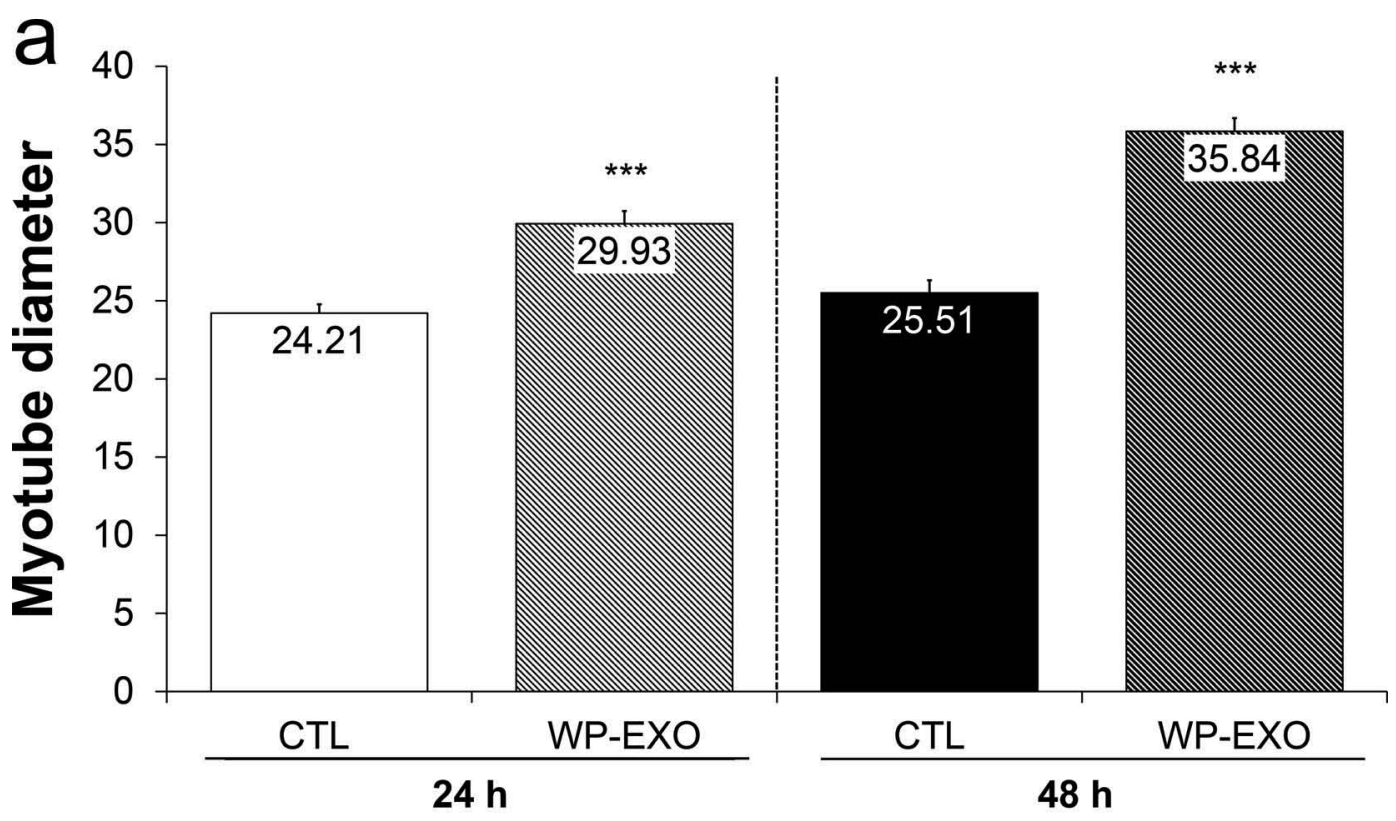

b
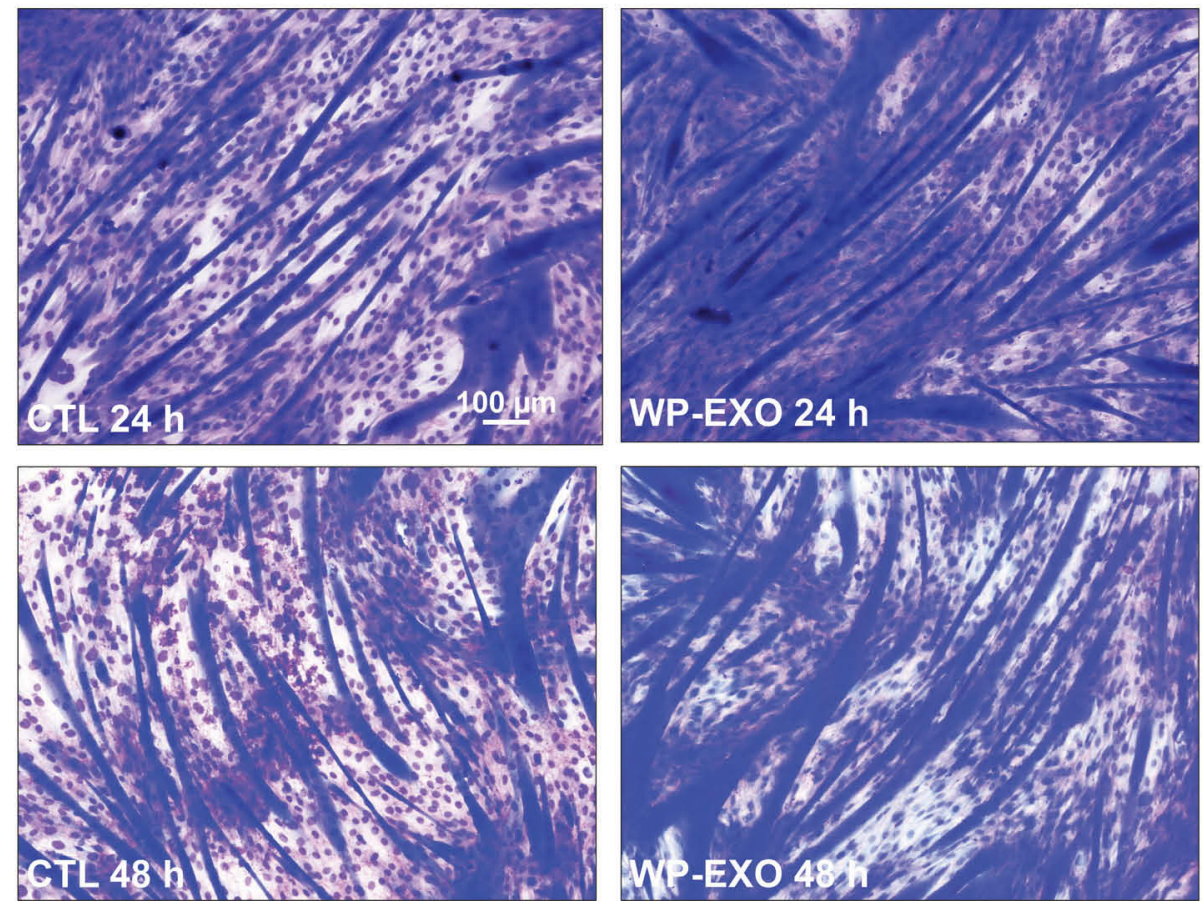

Figure 7. Effects of 24- and 48-h control (CTL) and whey protein-derived exosome (WP-EXO) treatments on myotube diameters ( $\mu$ m). (a) Myotube diameters from 30 myotubes per well (6 wells per treatment, means \pm SE) at each time point; (b) representative $10 \times$ digital bright field images of Jenner-Giemsa-stained myotubes. Independent $t$-tests were performed at each time point; asterisks indicate that WP-EXO was different from control $(* * * P<0.001)$. Color version available online. 
WHEY EXOSOMES AND MUSCLE PROTEIN SYNTHESIS

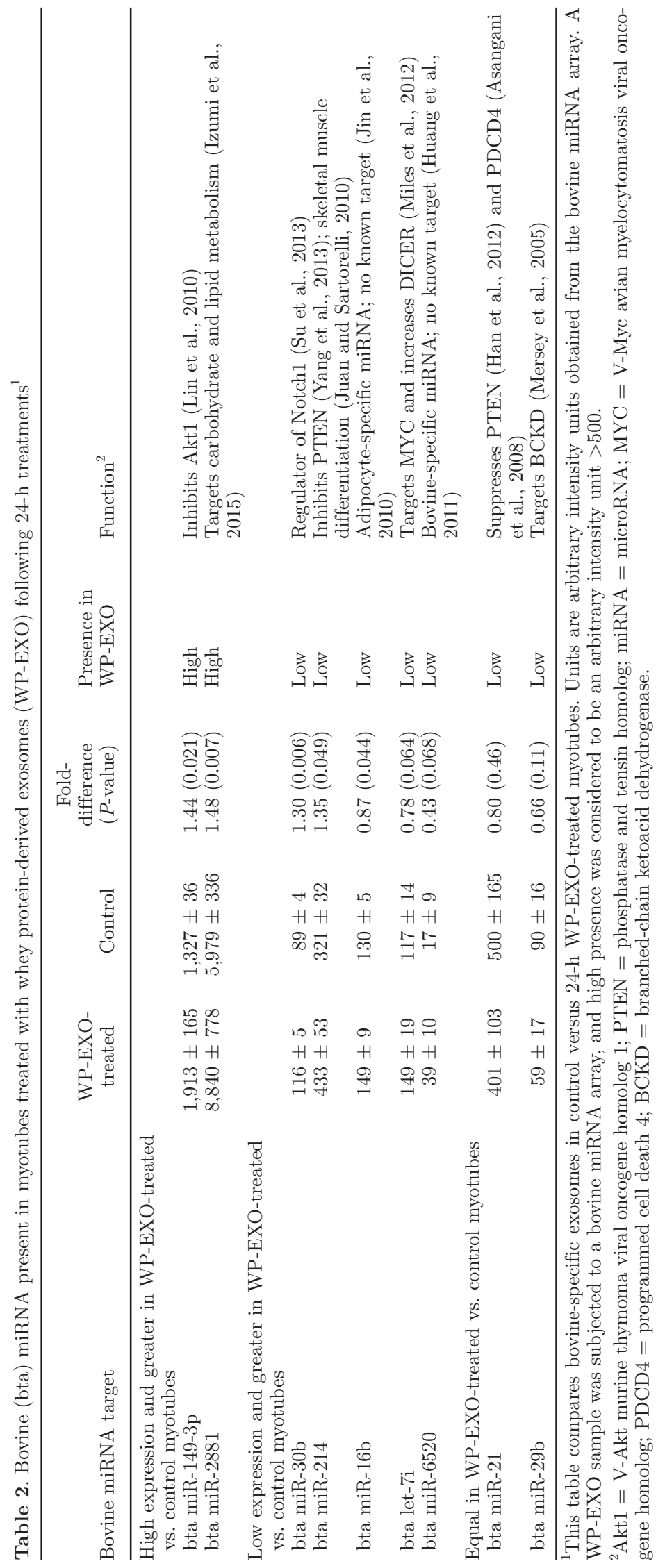

Journal of Dairy Science Vol. 100 No. 1, 2017 
increases in muscle mass in resistance-trained subjects (Cribb et al., 2006), and the amount of WP derived from milk supplementation $(3.5 \mathrm{~g} / \mathrm{d})$ in Hartman et al. (2007) is seemingly too low to promote an anabolic response relative to other data, suggesting that at least $20 \mathrm{~g}$ of protein is needed to elicit an MPS response (Moore et al., 2009). Again, this superior anabolic effect of milk consumption supplementation may have been due to the involvement of other bovine-derived bioactives present (i.e., bovine-derived exosomes or other unidentified compounds). In this regard, future in vivo studies will need to delineate the involvement of bovine milk- or WP-EXO and how (or even if) they can affect muscle anabolism.

In the present study, we demonstrated that WP elicited higher myotube MPS levels compared with EAA and multiple Leu doses, and found that (a) the EAA treatment matched the EAA profile of WP, and (b) all of the Leu treatments contained levels of Leu similar to or slightly higher than that in the WP treatments. Moreover, we demonstrated that, when LAT1 gene expression was knocked down in myotubes, myotube MPS levels were abolished to control levels in Leu-treated myotubes, whereas WP-treated myotubes exhibited an elevated response that was seemingly Leu-mediated, independent of mTOR signaling. It is curious to note, however, that although LAT1 mRNA expression was knocked down $40 \%$, protein levels were knocked down only $20 \%$ with our shRNA experiments. This finding may suggest that even a modest modulation of LAT1 protein levels may have profound effects on MPS levels. The LAT1 knockdown experiments were a critical aim in the current study, given that LAT1 is part of the plasma membrane transport L system integral to the uptake of large neutral amino acids (i.e., leucine and phenylalanine) into eukaryotic cells (Baird et al., 2009), and LAT1 gene expression in skeletal muscle is sensitive to mechanical loading and nutritional status (i.e., is increased in an mTOR-dependent fashion) (Drummond et al., 2012). Our data suggest that other "bioactive" components present in WP may be responsible for anabolism in a Leu-independent fashion.

Recent commentaries have posited that bovine milkderived exosomes possess miRNA that can target genes involved with skeletal MPS and are independent of mTOR signaling (Melnik et al., 2013; Melnik, 2015). Others have reported that isolation of WP from bovine milk leaves many of these exosomes intact (Hata et al., 2010; Yamada et al., 2012). Thus, we hypothesized that the Leu-independent anabolic effects of WP may have been a result of bovine miRNA present in WP-EXO. To isolate WP-EXO, we employed a series of centrifugation/filtering and reagent-based steps. Thereafter, we confirmed that exosomes existed in resuspended
WP powder through flow cytometry, Western blotting methods, and electron microscopy. Likewise, our bovine miRNA profiling confirmed that bovine-derived miRNA existed in the WP-EXO used to treat myotubes herein. Bovine-specific miRNA that have been posited to target genes related to hypertrophy (e.g., miR-149-3p, miR-21, miR-29b, miR-214) exist. Specifically, 2 of these (miR149-3p and miR-214) were enriched in the RNA pool of myotubes $24 \mathrm{~h}$ following WP-EXO treatments. Interestingly, miR-149-3p is an inhibitor of protein kinase B (Akt; Lin et al., 2010), and we observed 30 and $20 \%$ decreases in phospho-Akt protein expression in the 6- and $24 \mathrm{~h}$ WP-EXO-treated samples, respectively, suggesting that WP-EXO treatments may have facilitated this response through miRNA signaling. Additionally, miR214 has been shown to blunt the expression of PTEN, which could have positive effects on modulating mTOR to increase skeletal muscle hypertrophy (Melnik et al., 2013; Melnik, 2015) and the overexpression of miR-214 accelerates myotube differentiation (Lin et al., 2013). However, we did not observe a decrement in PTEN mRNA or protein expression, nor did we observe an increase in mTOR pathway markers in WP-EXO treatments. Hence, the $24 \mathrm{~h}$ post-treatment enrichment of miR-214 in WP-EXO-treated myotubes was apparently not a factor in promoting increases in MPS. Indeed, others have reported that rice protein (Zhang et al., 2012) contains miRNA that can affect gene expression in rodents within $6 \mathrm{~h}$ following feeding, so it remains plausible that our $24 \mathrm{~h}$ post-treatment miRNA analyses did not capture many of the transferred bovine miRNA that may have been enriched in WP-EXO-treated myotubes. Future research is needed that examines a more complete time course of bovine miRNA enrichment in myotubes treated from 3 to $24+$ hours, and the overexpression of bovine-specific miRNAs identified and how they affect myotube MPS and anabolism.

\section{CONCLUSIONS}

This study was not without limitations. First, all experiments in the present study were performed in vitro, and the findings require in vivo confirmation. Second, all studies were performed with growth or differentiation media that were not exosome-depleted. This was a major limitation, but we feel that this aspect of our study design added a bit of "real-world" complexity to our findings; specifically, if we relate our in vitro findings to humans, then it is presumable that humans also contain circulating exosomes. Thus, as in humans, we opted to use non-exosome-depleted media and sought to examine the biological effects that occur after enriching the media with hydrolyzed WP-EXO. The final limitation to this study from our perspective was 
the presumption that WP-EXO can be absorbed into circulation in vivo and exhibit their downstream physiological effects. Notwithstanding, this is the first study to demonstrate that WP contains exosomes capable of stimulating an anabolic response in skeletal muscle cells, independent of Leu-mediated mTOR signaling in vitro. Moreover, given that bovine-derived exosomes contain a wide species of miRNA and other biologically active components that can signal other tissues [e.g., adipose tissue and immune system (Juan and Sartorelli, 2010; Miles et al., 2012; Su et al., 2013; Yang et al., 2013; Izumi et al., 2015)], other physiological systems and their interaction with bovine-derived exosomes should be explored.

\section{ACKNOWLEDGMENTS}

We thank Rajesh H. Amin from the Auburn University Harrison School of Pharmacy for donating the $\mathrm{C}_{2} \mathrm{C}_{12}$ myoblasts used in these experiments. These studies were funded by laboratory startup funds provided by Michael D. Roberts (School of Kinesiology, Auburn University).

\section{REFERENCES}

Asangani, I. A., S. A. Rasheed, D. A. Nikolova, J. H. Leupold, N. H. Colburn, S. Post, and H. Allgayer. 2008. MicroRNA-21 (miR-21) post-transcriptionally downregulates tumor suppressor Pdcd4 and stimulates invasion, intravasation and metastasis in colorectal cancer. Oncogene 27:2128-2136.

Atherton, P. J., K. Smith, T. Etheridge, D. Rankin, and M. J. Rennie. 2010. Distinct anabolic signalling responses to amino acids in C2C12 skeletal muscle cells. Amino Acids 38:1533-1539.

Baier, S. R., C. Nguyen, F. Xie, J. R. Wood, and J. Zempleni. 2014. MicroRNAs are absorbed in biologically meaningful amounts from nutritionally relevant doses of cow milk and affect gene expression in peripheral blood mononuclear cells, HEK-293 kidney cell cultures, and mouse livers. J. Nutr. 144:1495-1500.

Baird, F. E., K. J. Bett, C. MacLean, A. R. Tee, H. S. Hundal, and P. M. Taylor. 2009. Tertiary active transport of amino acids reconstituted by coexpression of System A and L transporters in Xenopus oocytes. Am. J. Physiol. Endocrinol. Metab. 297:E822-E829.

Burd, N. A., H. M. Hamer, B. Pennings, W. F. Pellikaan, J. M. Senden, A. P. Gijsen, and L. J. van Loon. 2013. Substantial differences between organ and muscle specific tracer incorporation rates in a lactating dairy cow. PLoS One 8:e68109.

Chen, X., C. Gao, H. Li, L. Huang, Q. Sun, Y. Dong, C. Tian, S. Gao, H. Dong, D. Guan, X. Hu, S. Zhao, L. Li, L. Zhu, Q. Yan, J. Zhang, K. Zen, and C. Y. Zhang. 2010. Identification and characterization of microRNAs in raw milk during different periods of lactation, commercial fluid, and powdered milk products. Cell Res. 20:1128-1137.

Cribb, P. J., A. D. Williams, M. F. Carey, and A. Hayes. 2006. The effect of whey isolate and resistance training on strength, body composition, and plasma glutamine. Int. J. Sport Nutr. Exerc. Metab. 16:494-509.

Deldicque, L., C. Sanchez Canedo, S. Horman, I. De Potter, L. Bertrand, L. Hue, and M. Francaux. 2008. Antagonistic effects of leucine and glutamine on the mTOR pathway in myogenic $\mathrm{C} 2 \mathrm{C} 12$ cells. Amino Acids 35:147-155.

Drummond, M. J., J. M. Dickinson, C. S. Fry, D. K. Walker, D. M. Gundermann, P. T. Reidy, K. L. Timmerman, M. M. Markof- ski, D. Paddon-Jones, B. B. Rasmussen, and E. Volpi. 2012. Bed rest impairs skeletal muscle amino acid transporter expression, mTORC1 signaling, and protein synthesis in response to essential amino acids in older adults. Am. J. Physiol. Endocrinol. Metab. 302:E1113-E1122.

Ha, E., and M. B. Zemel. 2003. Functional properties of whey, whey components, and essential amino acids: mechanisms underlying health benefits for active people. J. Nutr. Biochem. 14:251-258. (review).

Han, M., M. Liu, Y. Wang, X. Chen, J. Xu, Y. Sun, L. Zhao, H. Qu, Y. Fan, and C. Wu. 2012. Antagonism of miR-21 reverses epithelial-mesenchymal transition and cancer stem cell phenotype through AKT/ERK1/2 inactivation by targeting PTEN. PLoS One $7: \mathrm{e} 39520$.

Hartman, J. W., J. E. Tang, S. B. Wilkinson, M. A. Tarnopolsky, R. L. Lawrence, A. V. Fullerton, and S. M. Phillips. 2007. Consumption of fat-free fluid milk after resistance exercise promotes greater lean mass accretion than does consumption of soy or carbohydrate in young, novice, male weightlifters. Am. J. Clin. Nutr. 86:373-381.

Hata, T., K. Murakami, H. Nakatani, Y. Yamamoto, T. Matsuda, and N. Aoki. 2010. Isolation of bovine milk-derived microvesicles carrying mRNAs and microRNAs. Biochem. Biophys. Res. Commun. 396:528-533.

Huang, J., Z. Ju, Q. Li, Q. Hou, C. Wang, J. Li, R. Li, L. Wang, T. Sun, S. Hang, Y. Gao, M. Hou, and J. Zhong. 2011. Solexa sequencing of novel and differentially expressed microRNAs in testicular and ovarian tissues in Holstein cattle. Int. J. Biol. Sci. 7:1016-1026.

Hulmi, J. J., C. M. Lockwood, and J. R. Stout. 2010. Effect of protein/ essential amino acids and resistance training on skeletal muscle hypertrophy: A case for whey protein. Nutr. Metab. (Lond.) 7:51.

Izumi, H., N. Kosaka, T. Shimizu, K. Sekine, T. Ochiya, and M. Takase. 2012. Bovine milk contains microRNA and messenger RNA that are stable under degradative conditions. J. Dairy Sci. 95:4831-4841.

Izumi, H., N. Kosaka, T. Shimizu, K. Sekine, T. Ochiya, and M. Takase. 2013. Purification of RNA from milk whey. Methods Mol. Biol. 1024:191-201.

Izumi, H., M. Tsuda, Y. Sato, N. Kosaka, T. Ochiya, H. Iwamoto, K. Namba, and Y. Takeda. 2015. Bovine milk exosomes contain microRNA and mRNA and are taken up by human macrophages. J. Dairy Sci. 98:2920-2933.

Jin, W., M. V. Dodson, S. S. Moore, J. A. Basarab, and L. L. Guan. 2010. Characterization of microRNA expression in bovine adipose tissues: A potential regulatory mechanism of subcutaneous adipose tissue development. BMC Mol. Biol. 11:29.

Juan, A. H., and V. Sartorelli. 2010. MicroRNA-214 and polycomb group proteins: A regulatory circuit controlling differentiation and cell fate decisions. Cell Cycle 9:1445-1446.

Kimball, S. R., L. M. Shantz, R. L. Horetsky, and L. S. Jefferson. 1999. Leucine regulates translation of specific mRNAs in L6 myoblasts through mTOR-mediated changes in availability of eIF4E and phosphorylation of ribosomal protein S6. J. Biol. Chem. 274:11647-11652.

Lässer, C., M. Eldh, and J. Lotvall. 2012. Isolation and characterization of RNA-containing exosomes. J. Vis. Exp. 59:e3037.

Latres, E., A. R. Amini, A. A. Amini, J. Griffiths, F. J. Martin, Y. Wei, H. C. Lin, G. D. Yancopoulos, and D. J. Glass. 2005. Insulinlike growth factor-1 (IGF-1) inversely regulates atrophy-induced genes via the phosphatidylinositol 3-kinase/Akt/mammalian target of rapamycin (PI3K/Akt/mTOR) pathway. J. Biol. Chem. 280:2737-2744.

Lin, R. J., Y. C. Lin, and A. L. Yu. 2010. miR-149* induces apoptosis by inhibiting Akt1 and E2F1 in human cancer cells. Mol. Carcinog. 49:719-727.

Lin, Y., Y. Zeng, F. Zhang, L. Xue, Z. Huang, W. Li, and M. Guo. 2013. Characterization of microRNA expression profiles and the discovery of novel microRNAs involved in cancer during human embryonic development. PLoS One 8:e69230.

Liu, X., B. Fu, D. Chen, Q. Hong, J. Cui, J. Li, X. Bai, and X. Chen. 2015. miR-184 and miR-150 promote renal glomerular me- 
sangial cell aging by targeting Rab1a and Rab31. Exp. Cell Res. 336:192-203.

McGinnis, G. R., C. Ballmann, B. Peters, G. Nanayakkara, M. Roberts, R. Amin, and J. C. Quindry. 2015. Interleukin-6 mediates exercise preconditioning against myocardial ischemia reperfusion injury. Am. J. Physiol. Heart Circ. Physiol. 308:H1423-H1433.

Melnik, B. C. 2015. Milk-A nutrient system of mammalian evolution promoting mTORC1-dependent translation. Int. J. Mol. Sci. 16:17048-17087.

Melnik, B. C., S. M. John, and G. Schmitz. 2013. Milk is not just food but most likely a genetic transfection system activating mTORC1 signaling for postnatal growth. Nutr. J. 12:103.

Mersey, B. D., P. Jin, and D. J. Danner. 2005. Human microRNA (miR29b) expression controls the amount of branched chain alpha-ketoacid dehydrogenase complex in a cell. Hum. Mol. Genet. 14:3371-3377.

Miles, J. R., T. G. McDaneld, R. T. Wiedmann, R. A. Cushman, S. E. Echternkamp, J. L. Vallet, and T. P. Smith. 2012. MicroRNA expression profile in bovine cumulus-oocyte complexes: possible role of let-7 and miR-106a in the development of bovine oocytes. Anim. Reprod. Sci. 130:16-26.

Mobley, C. B., C. D. Fox, B. S. Ferguson, R. H. Amin, V. J. Dalbo, S. Baier, J. A. Rathmacher, J. M. Wilson, and M. D. Roberts. 2014. L-leucine, beta-hydroxy-beta-methylbutyric acid (HMB) and creatine monohydrate prevent myostatin-induced Akirin-1/Mighty mRNA down-regulation and myotube atrophy. J. Int. Soc. Sports Nutr. 11:38.

Mobley, C. B., C. D. Fox, B. S. Ferguson, C. A. Pascoe, J. C. Healy, J. S. McAdam, C. M. Lockwood, and M. D. Roberts. 2015. Effects of protein type and composition on postprandial markers of skeletal muscle anabolism, adipose tissue lipolysis, and hypothalamic gene expression. J. Int. Soc. Sports Nutr. 12:14.

Mobley, C. B., C. D. Fox, R. M. Thompson, J. C. Healy, V. Santucci, W. C. Kephart, A. E. McCloskey, M. Kim, D. D. Pascoe, J. S. Martin, J. R. Moon, K. C. Young, and M. D. Roberts. 2016. Comparative effects of whey protein versus L-leucine on skeletal muscle protein synthesis and markers of ribosome biogenesis following resistance exercise. Amino Acids 48:733-750.

Moore, D. R., M. J. Robinson, J. L. Fry, J. E. Tang, E. I. Glover, S. B. Wilkinson, T. Prior, M. A. Tarnopolsky, and S. M. Phillips. 2009. Ingested protein dose response of muscle and albumin protein synthesis after resistance exercise in young men. Am. J. Clin. Nutr. 89:161-168.

Peyrollier, K., E. Hajduch, A. S. Blair, R. Hyde, and H. S. Hundal. 2000. L-leucine availability regulates phosphatidylinositol 3-kinase, p70 S6 kinase and glycogen synthase kinase-3 activity in L6 muscle cells: Evidence for the involvement of the mammalian target of rapamycin (mTOR) pathway in the L-leucine-induced up-regulation of system A amino acid transport. Biochem. J. 350:361-368.

Phillips, S. M., J. E. Tang, and D. R. Moore. 2009. The role of milkand soy-based protein in support of muscle protein synthesis and muscle protein accretion in young and elderly persons. J. Am. Coll. Nutr. 28:343-354.

Reinhardt, T. A., J. D. Lippolis, B. J. Nonnecke, and R. E. Sacco. 2012. Bovine milk exosome proteome. J. Proteomics 75:1486-1492.

Roberts, M. D., C. L. Cruthirds, C. M. Lockwood, K. Pappan, T. E. Childs, J. M. Company, J. D. Brown, R. G. Toedebusch, and F. W. Booth. 2014. Comparing serum responses to acute feedings of an extensively hydrolyzed whey protein concentrate versus a native whey protein concentrate in rats: A metabolomics approach. Appl. Physiol. Nutr. Metab. 39:158-167.
Roberts, M. D., A. M. Holland, W. C. Kephart, C. B. Mobley, P. W. Mumford, R. P. Lowery, C. D. Fox, A. E. McCloskey, J. J. Shake, P. Mesquita, R. K. Patel, J. S. Martin, K. C. Young, A. N. Kavazis, and J. M. Wilson. 2015. A putative low-carbohydrate ketogenic diet elicits mild nutritional ketosis but does not impair the acute or chronic hypertrophic responses to resistance exercise in rodents. J. Appl. Physiol. 120:1173-1185. https://doi.org/10.1152/ japplphysiol.00837.2015.

Smith, L. A., and P. X. Ma. 2004. Nano-fibrous scaffolds for tissue engineering. Colloids Surf. B Biointerfaces 39:125-131.

Stec, M. J., N. A. Kelly, G. M. Many, S. T. Windham, S. C. Tuggle, and M. M. Bamman. 2016. Ribosome biogenesis may augment resistance training-induced myofiber hypertrophy and is required for myotube growth in vitro. Am. J. Physiol. Endocrinol. Metab. 310:E652-E661. https://doi.org/10.1152/ajpendo.00486.2015.

Stitt, T. N., D. Drujan, B. A. Clarke, F. Panaro, Y. Timofeyva, W. O. Kline, M. Gonzalez, G. D. Yancopoulos, and D. J. Glass. 2004. The IGF-1/PI3K/Akt pathway prevents expression of muscle atrophy-induced ubiquitin ligases by inhibiting FOXO transcription factors. Mol. Cell 14:395-403.

Su, X., C. Qian, Q. Zhang, J. Hou, Y. Gu, Y. Han, Y. Chen, M. Jiang, and X. Cao. 2013. miRNomes of haematopoietic stem cells and dendritic cells identify miR-30b as a regulator of Notch1. Nat. Commun. 4:2903.

Tang, J. E., D. R. Moore, G. W. Kujbida, M. A. Tarnopolsky, and S. M. Phillips. 2009. Ingestion of whey hydrolysate, casein, or soy protein isolate: effects on mixed muscle protein synthesis at rest and following resistance exercise in young men. J. Appl. Physiol. 107:987-992.

Tracz, E., D. W. Dickson, J. F. Hainfeld, and H. Ksiezak-Reding. 1997. Paired helical filaments in corticobasal degeneration: the fine fibrillary structure with NanoVan. Brain Res. 773:33-44.

van Vliet, S., N. A. Burd, and L. J. van Loon. 2015. The skeletal muscle anabolic response to plant- versus animal-based protein consumption. J. Nutr. 145:1981-1991.

Veliça, P., and C. M. Bunce. 2011. A quick, simple and unbiased method to quantify $\mathrm{C} 2 \mathrm{C} 12$ myogenic differentiation. Muscle Nerve 44:366-370.

Wang, X., L. E. Campbell, C. M. Miller, and C. G. Proud. 1998. Amino acid availability regulates p70 S6 kinase and multiple translation factors. Biochem. J. 334:261-267.

Wolf, T., S. R. Baier, and J. Zempleni. 2015. The intestinal transport of bovine milk exosomes is mediated by endocytosis in human colon carcinoma Caco-2 cells and rat small intestinal IEC-6 cells. J. Nutr. 145:2201-2206.

Yamada, T., Y. Inoshima, T. Matsuda, and N. Ishiguro. 2012. Comparison of methods for isolating exosomes from bovine milk. J. Vet. Med. Sci. 74:1523-1525.

Yang, T. S., X. H. Yang, X. D. Wang, Y. L. Wang, B. Zhou, and Z. S. Song. 2013. MiR-214 regulate gastric cancer cell proliferation, migration and invasion by targeting PTEN. Cancer Cell Int. 13:68

Zhang, L., D. Hou, X. Chen, D. Li, L. Zhu, Y. Zhang, J. Li, Z. Bian, X. Liang, X. Cai, Y. Yin, C. Wang, T. Zhang, D. Zhu, D. Zhang, J. Xu, Q. Chen, Y. Ba, J. Liu, Q. Wang, J. Chen, J. Wang, M. Wang, Q. Zhang, J. Zhang, K. Zen, and C. Y. Zhang. 2012. Exogenous plant MIR168a specifically targets mammalian LDLRAP1 evidence of cross-kingdom regulation by microRNA. Cell Res. $22: 107-126$ 